\title{
On the Expressiveness of MTL Variants over Dense Time
}

\author{
Carlo A. Furia and Matteo Rossi
}

May 2007

\begin{abstract}
The basic modal operator bounded until of Metric Temporal Logic (MTL) comes in several variants. In particular it can be strict (when it does not constrain the current instant) or not, and matching (when it requires its two arguments to eventually hold together) or not. This paper compares the relative expressiveness of the resulting MTL variants over dense time. We prove that the expressiveness is not affected by the variations when considering non-Zeno interpretations and arbitrary nesting of temporal operators. On the contrary, the expressiveness changes for flat (i.e., without nesting) formulas, or when Zeno interpretations are allowed.
\end{abstract}




\section{Contents}

\begin{tabular}{lll}
\hline & Introduction & 3
\end{tabular}

2 MTL and Its Variants $\quad 6$

2.1 Syntax . . . . . . . . . . . . . . . . . . . . . 6

2.2 Semantics . . . . . . . . . . . . . . . 7

2.2.1 Non-Zenoness . . . . . . . . . . . . . . . 8

3 Nesting MTL over non-Zeno Behaviors 9

3.1 Non-Strict as Expressive as Strict . . . . . . . . . . . . . . . . . . 9

3.2 Matching as Expressive as Non-Matching . . . . . . . . . . . . . 10

4 Flat MTL 11

4.1 Non-Strict Less Expressive Than Strict . . . . . . . . . . . . . . . 11

$4.1 .1 \quad$ Initial Satisfiability . . . . . . . . . . . . . . . . . . . . 12

4.1 .2 Global Satisfiability . . . . . . . . . . . . . . . 13

4.2 Matching Less Expressive Than Non-Matching . . . . . . . . . . 16

4.3 Non-Strict Matching Less Expressive Than Matching . . . . . . . 17

$4.3 .1 \quad$ Initial Satisfiability . . . . . . . . . . . . . . 17

4.3.2 Global Satisfiability . . . . . . . . . . . . . 18

4.4 Non-Strict Matching Less Expressive Than Non-Strict . . . . . . 19

$4.4 .1 \quad$ Initial Satisfiability . . . . . . . . . . . . . . . 19

4.4 .2 Global Satisfiability . . . . . . . . . . . . . . . . 20

4.5 Flat Less Expressive Than Nesting . . . . . . . . . . . . . . . . . 21

4.5.1 Initial Satisfiability . . . . . . . . . . . . . . 21

4.5.2 Global Satisfiability . . . . . . . . . . . . . 22

5 Nesting MTL over Zeno Behaviors $\quad 25$

5.1 Non-Strict Less Expressive Than Strict. . . . . . . . . . . . . . . 25

$5.1 .1 \quad$ Primitive Behavior of MTL Formulas . . . . . . . . . . . 26

5.1 .2 Non-Strict Is Less Expressive Than Strict . . . . . . . . . 30

5.2 Non-Strict Matching Less Expressive Than Strict Matching . . . 30

5.3 Matching as Expressive as Non-Matching . . . . . . . . . . . . . 31

5.3 .1 Strict Variant . . . . . . . . . . . . . . . . . 31

5.3.2 Non-Strict Variant . . . . . . . . . . . . . . . . . . . 32

6 Summary and Discussion 34 


\section{Introduction}

In the last few decades, the formal description and analysis of real-time systems has become an increasingly important research topic. This has resulted, among other things, in the development of several formal notations for the description of real-time properties and systems. In particular, a significant number of extensions of classical temporal logics to deal with metric (quantitative) time has been introduced and used (see e.g., [AH92b, AH93, FMMR07]). Among them, Metric Temporal Logic (MTL) [Koy90, AH93] is one of the most popular. An appealing feature of MTL is its being a straightforward extension of well-known Linear Temporal Logic (LTL), a classical temporal logic. In MTL, an interval parameter is added to LTL's modal operators (such as the until operator). The interval specifies a range of distances over which the arguments of the modality must hold, thus allowing the expression of real-time properties.

MTL formulas can be interpreted over a variety of temporal models; in particular both discrete (e.g., $\mathbb{N}, \mathbb{Z}$ ) and dense (e.g., $\mathbb{R}$ ) time domains are possible. Metric operators over discrete time can be regarded as simple "syntactic sugar", i.e., as a succinct way of expressing metric constraints that are however expressible with LTL's next modality. This entails that all well-known techniques and results about the expressiveness of LTL and its variants [Eme90] can often be "lifted" to the real-time case [AH93]. On the contrary, the analysis for dense-time models usually requires novel techniques, as additional difficulties and complications are commonly encountered. In fact, after Alur and Henzinger's seminal work [AH93], only in more recent years a detailed expressiveness analysis of MTL over dense-time domains is being carried out (e.g., [HR04, HR06, BCM05, DP07, PD06, OW07, BMOW07]).

Another aspect where the use of MTL (and temporal logics in general) over metric dense-time models shows a substantial difference with respect to discrete time is in the robustness of the language expressiveness with respect to changes in its (syntactic) definitions or in the choice of the underlying interpretation structures. In other words, it is often the case that apparently minimal changes in the definition of the basic modal operators, or in the choice of the interpretation structures (e.g., timed words rather than timed interval sequences), of the logics yield substantial differences in the resulting expressiveness. Also, these differences are usually more difficult to predict and assess than in the discretetime case. One significant example is the use of a "natural" extension such as the introduction of past operators: it is well-known that adding them does not change the expressive power over discrete time [GPSS80, Eme90] (while it increases the succinctness [LMS02]), but it does over dense time for both LTL [GHR94, HR03] and MTL over various models [BCM05, PD06] (Alur and Henzinger [AH92a] were the first to analyze this issue for an MTL subset known as MITL [AFH96]).

This paper contributes to enriching the emerging picture about the expressiveness of MTL and its common variants. The reference interpretation structure is the behavior, that is generic mappings that associate with every instant of time the propositions that are true at that instant. When behaviors are restricted to be non-Zeno [GM01, AL94] (also called finitely variable [Rab03, HR04]) they are an equivalent way of expressing the well-known timed interval sequences [Asa04]. We consider two basic language features to be varied in MTL definitions: strictness and matchingness. The basic until operator 
$\mathrm{U}\left(\phi_{1}, \phi_{2}\right)$ is called strict (in its first argument) if it does not constrain its first argument $\phi_{1}$ to hold at the current instant (i.e., it constraints strictly the future); on the other hand the same operator is called matching if it requires the second argument $\phi_{2}$ to hold together with the first argument $\phi_{1}$ at some instant in the future. The most common MTL definition uses an until operator that is strict and non-matching; it is simple to realize that this does not restrict the expressiveness as the matching and non-strict untils are easily expressible in terms of strict non-matching untils. However, some applications dealing with MTL or closely related languages (e.g., [MNP05, MNP06, FR06]) are based on the matching or non-strict variants, or both. Therefore it is interesting to analyze if these syntactic restrictions imply restrictions in the expressiveness of the language.

Another dimension that we consider in our analysis is the restriction to flat MTL formulas, i.e., formulas that do not nest temporal operators. These have also been used, among others, in some previous work of ours [FR06], as well as in several works with classical (qualitative) temporal logic (see "Related work" sub-section). It is an easy guess that flat MTL is less expressive than its full "nesting" counterpart; in this paper we prove this intuition and then we analyze how the relationships between the various (non-)matching and (non)strict variants change when restricted to flat formulas. In order to do so, we develop techniques to handle two different definitions in the satisfaction semantics of formulas: initial satisfiability — where the truth of a formula is evaluated only at some initial time (typically 0) — and global satisfiability - where the truth of a formula is evaluated at all time instants. While the two semantics are easily reconcilable when nesting is allowed, passing from the initial semantics to the global one with flat formulas is more challenging and requires some ingenuity. We consider also the less common global satisfiability semantics because the expressiveness of the flat fragment is non-trivial under such semantics (in fact, it corresponds to an implicit nesting of a qualitative temporal operator), and most common real-time properties such as bounded response and bounded invariance [Koy90] can be easily expressed.

Finally, we also consider what happens to (relative) expressiveness if Zeno behaviors are allowed. In particular, we show that some equivalences between MTL variations that hold over non-Zeno behaviors are no more valid with Zeno behaviors. In this sense, allowing Zeno behaviors weakens the robustness of MTL expressiveness with respect to definitions, and it renders the picture more complicated and less intuitive.

Related works. As mentioned above, in recent years several works have analyzed the expressiveness of different MTL variants over dense time. Let us recall briefly the main results of a significant subset thereof.

Bouyer et al. [BCM05] compare the expressiveness of MTL with that of TPTL (another real-time temporal logic [AH94]) and they are the first to prove the conjecture that the latter is strictly more expressive than the former, over both timed words and timed interval sequences. As a corollary of their results, they show that past operators increase the expressiveness of MTL over both interpretation structures.

Since the work of Alur and Henzinger [AH93] it is known that (full) MTL is undecidable over dense-time models. This shortcoming has been long at- 
tributed to the possibility of expressing punctual (i.e., exact) timing constraints; in fact Alur et al. AFH96] have shown that MITL, a MTL subset where punctual intervals are disallowed, is decidable. However, punctuality does not always entails undecidability. In fact, Ouaknine and Worrel [OW07] have been the first to prove that MTL is decidable over finite timed words, albeit with non-primitive recursive complexity; their proofs rely on automata-based techniques, and in particular on the notion of timed automata with alternation [LW05]. On the other hand, they show that several significant fragments of MTL are still undecidable over infinite timed words. In the same vein, Bouyer et al. [BMOW07] have identified significant MTL fragments that are instead decidable (with primitive complexity) even if one allows the expression of punctual timing constraints.

D'Souza and Prabhakar [DP07] compare the expressiveness of MTL over the two interpretation structures of timed words and timed interval sequences (more precisely, a specialization of the latter called "continuous" semantics). Building upon Ouaknine and Worrel's decidability results for MTL [OW07], they show that MTL is strictly more expressive over timed interval sequences than it is over timed words. The same authors [PD06] analyze a significant number of MTL variations, namely those obtained by adding past operators or by considering qualitative operators rather than metric ones, over both timed words and timed interval sequences, both in their finite and infinite forms. Still the same authors [DMP07] have shown how to rewrite MTL formulas in flat form, and without past operators, by introducing additional propositions (a similar flattening has been shown for another temporal logic in [Fur07]). While these latter results do not pertain directly to the expressiveness of the language (because of the new propositions that are introduced) they help assessing the decidability of MTL variations.

In previous work [FR07a] we proved the equivalence between the strict and non-strict non-matching variants of MTL over non-Zeno behaviors and with arbitrarily nested formulas. Section 3 uses techniques similar to those in [FR07a] to prove new equivalence results.

All these articulated results, that we do not report with further details for the lack of space, show how relative expressiveness relations are much more complicated over dense time than they are over discrete time. In fact, some authors (e.g., [Asa04, HR04, AH92b]) have suggested that these additional difficulties are an indication that the "right" semantic model for dense time has not been found yet. In particular, Hirshfeld and Rabinovich [HR04, HR06] have made a strong point that most approaches to the definition of temporal logics for real-time and to their semantics depart from the "classical" approach to temporal logic and are too ad hoc, which results in unneeded complexity and lack of robustness. While we agree with several of their remarks, we must also acknowledge that MTL (and other similar logics) has become a popular notation, and it has been used in several works. As a consequence, it is important to assess precisely the expressiveness of the language and of its common variants because of the impact on the scope of those works, even if focusing on different languages might have opened the door to more straightforward approaches.

Finally, let us mention that several works dealing with classical (qualitative) temporal logic considered variants in the definition of the basic modalities, and their impact on expressiveness and complexity. Let us mention the work by Demri and Schnoebelen [DS02] where the complexity of LTL without nesting, 
or with a bounded nesting depth, over discrete time is thoroughly investigated. Also, several works have given a very detailed characterization of how the expressiveness of LTL varies with the number of nested modalities [EW96, TW04, KS05]; and several other works, such as [MC85, DG99, Dam99, CC00], have used and characterized a flat fragment of LTL where nesting is only allowed in the second argument of any until formula.

Reynolds [Rey03] has proved that, over dense time, LTL with strict until is strictly more expressive than LTL with a variant of non-strict until which includes the current instant. Note that Reynold's non-strict until has a different (weaker) semantics than the one we consider in this paper, because of the restriction to include the current instant. In other words, according to the notation that we are introducing in Section 2, [Rey03] compares the strict $\widetilde{U}_{(0,+\infty)}$ to the non-strict $\mathrm{U}_{[0,+\infty)}$; as a consequence, Reynold's result is orthogonal to ours.

Outline. The paper is organized as follows. Section 2 presents the syntax and semantics of MTL and its variants over dense time; Section 3 presents proofs that all these variants are equally expressive over non-Zeno behaviors, for MTL formulas with arbitrary nesting; on the contrary, Section 4 considers flat MTL formulas and proves several separation results between the previously introduced variants; Section 5 reconsiders the MTL nesting formulas of Section 3 and shows that the equivalence results no longer holds over Zeno behaviors. Finally, Section 6 summarizes the expressiveness results of the paper and outlines future work.

\section{MTL and Its Variants}

This section defines the syntax and semantics of MTL and its variants over dense-time models.

\subsection{Syntax}

MTL is built out of the single modal operator bounded unti $i^{1}$ through propositional composition. Formulas are built as follows, where $I$ is an interval $\langle l, u\rangle$ of the reals such that $0 \leq l \leq u \leq+\infty$ and $l \in \mathbb{Q}, u \in \mathbb{Q} \cup\{+\infty\}$, and $\mathrm{p} \in \mathcal{P}$ is some atomic proposition from a finite set $\mathcal{P}$.

$$
\phi \quad::=\mathrm{p}\left|\widetilde{\mathrm{U}}_{I}\left(\phi_{1}, \phi_{2}\right)\right| \neg \phi \mid \phi_{1} \wedge \phi_{2}
$$

The tilde above the $\widetilde{U}_{I}$ of the until operator denotes that it is strict, as it will be apparent in the definition of its semantics; $\widetilde{U}_{I}$ is also meant to be nonmatching. We denote the set of formulas generated by the rule above as $\widetilde{\mathrm{MTL}}$, which is therefore strict non-matching.

From the basic strict operator we can define syntactically some variants: the non-strict non-matching until $\mathrm{U}_{I}$, the strict matching until $\widetilde{\mathbf{U}}_{I}^{\downarrow}$, and the non-strict matching until $\bigcup_{I}^{\downarrow}$. They are defined in Table 1, together with a few other derived modal operators of common use; derived propositional connectives (such as $\Rightarrow, \vee, \Leftrightarrow$ ) are defined as usual. For derived operators we use the same

\footnotetext{
${ }^{1}$ In this paper we consider MTL with future operators only.
} 
notational conventions, i.e., a tilde denotes strictness and a $\downarrow$ denotes matchingness. Accordingly, we denote by MTL the set of non-strict non-matching formulas (i.e., those using only the $U_{I}$ operator), by $\widetilde{\mathrm{MTL}}{ }^{\downarrow}$ the set of strict matching formulas (i.e., those using only the $\widetilde{U}_{I}^{\downarrow}$ operator), and by MTL ${ }^{\downarrow}$ the set of non-strict matching formulas (i.e., those using only the $\mathrm{U}_{I}^{\downarrow}$ operator). The same notational convention is used for derived operators Also, we adopt the convention of dropping the interval $I$ when it is $(0,+\infty)$, and to denote punctual intervals (i.e., intervals of the form $[d, d]$ for some $d$ ) with the abbreviation $=d$.

\begin{tabular}{|ccc|}
\hline OPERATOR & $\equiv$ & DEFINITION \\
\hline $\mathrm{U}_{I}\left(\phi_{1}, \phi_{2}\right)$ & $\equiv$ & if $0 \notin I: \phi_{1} \wedge \widetilde{\mathrm{U}}_{I}\left(\phi_{1}, \phi_{2}\right)$ else: $\phi_{2} \vee\left(\phi_{1} \wedge \widetilde{\mathrm{U}}_{I}\left(\phi_{1}, \phi_{2}\right)\right)$ \\
$\widetilde{\mathrm{U}}_{I}^{\downarrow}\left(\phi_{1}, \phi_{2}\right)$ & $\equiv$ & if $0 \notin I: \widetilde{\mathrm{U}}_{I}\left(\phi_{1}, \phi_{2} \wedge \phi_{1}\right)$ else: $\phi_{2} \vee\left(\widetilde{\mathrm{U}}_{I}\left(\phi_{1}, \phi_{2} \wedge \phi_{1}\right)\right)$ \\
$\mathrm{U}_{I}^{\downarrow}\left(\phi_{1}, \phi_{2}\right)$ & $\equiv$ & $\phi_{1} \wedge \widetilde{\mathbf{U}}_{I}\left(\phi_{1}, \phi_{2} \wedge \phi_{1}\right) \equiv \mathrm{U}_{I}\left(\phi_{1}, \phi_{2} \wedge \phi_{2}\right)$ \\
$\widetilde{\mathrm{R}}_{I}^{\downarrow}\left(\phi_{1}, \phi_{2}\right)$ & $\equiv$ & $\widetilde{\mathrm{U}}_{I}^{\downarrow}\left(\neg \phi_{1}, \neg \phi_{2}\right)$ \\
$\mho_{I}(\phi)$ & $\equiv$ & $\widetilde{\mathrm{U}}_{I}(\top, \phi)$ \\
$\square_{I}(\phi)$ & $\equiv$ & $\neg \diamond_{I}(\neg \phi)$ \\
$\bigcirc(\phi)$ & $\equiv$ & $\mathrm{U}_{(0,+\infty)}(\phi, \top)$ \\
$\widetilde{\bigcirc}(\phi)$ & $\equiv$ & $\widetilde{\mathrm{U}}_{(0,+\infty)}(\phi, \top)$ \\
\hline
\end{tabular}

Table 1: MTL derived temporal operators

Granularity and nesting depth. The granularity $\rho$ of a formula $\phi$ is defined as the reciprocal of the product of all denominators of non-null finite interval bounds appearing in $\phi$. More formally, it is defined by the following recursive expression.

$$
\rho(\phi)= \begin{cases}1 & \text { if } \phi=\mathrm{p} \\ \rho(\psi) & \text { if } \phi=\neg \psi \\ \rho\left(\psi_{1}\right) \cdot \rho\left(\psi_{2}\right) & \text { if } \phi=\psi_{1} \wedge \psi_{2} \text { or } \phi=\widetilde{\mathrm{U}}_{\langle 0, \infty\rangle}\left(\psi_{1}, \psi_{2}\right) \\ \frac{1}{L} \cdot \frac{1}{U} \cdot \rho\left(\psi_{1}\right) \cdot \rho\left(\psi_{2}\right) & \text { if } \phi=\widetilde{\mathrm{U}}_{\langle l / L, u / U\rangle}\left(\psi_{1}, \psi_{2}\right) \text { and } l, u \neq 0,+\infty \\ \frac{1}{L} \cdot \rho\left(\psi_{1}\right) \cdot \rho\left(\psi_{2}\right) & \text { if } \phi=\widetilde{\mathbb{U}}_{\langle l / L,+\infty\rangle}\left(\psi_{1}, \psi_{2}\right) \text { and } l \neq 0 \\ \frac{1}{U} \cdot \rho\left(\psi_{1}\right) \cdot \rho\left(\psi_{2}\right) & \text { if } \phi=\widetilde{\mathbb{U}}_{\langle 0, u / U\rangle}\left(\psi_{1}, \psi_{2}\right) \text { and } u \neq+\infty\end{cases}
$$

The nesting depth (also called temporal height) of a formula $\phi$ is defined as the maximum number of nested modalities. A formula is called flat if it does not nest modal operators, and nesting otherwise. Given a set of formulas $F$, the subset of all its flat formulas is denoted by bF (for instance flat non-strict non-matching formulas are denoted as bMTL).

\subsection{Semantics}

We define formally the semantics of $\widetilde{\text { MTL }}$ over generic Boolean behaviors. Given a time domain $\mathbb{T}$ and a finite set of atomic propositions $\mathcal{P}$, a Boolean behavior over $\mathcal{P}$ is a mapping $b: \mathbb{T} \rightarrow 2^{\mathcal{P}}$ from the time domain to subsets of $\mathcal{P}$ : for every time instant $t \in \mathbb{T}, b$ maps $t$ to the set of propositions $b(t)$ that are true at $t$. We denote the set of all mappings for a given set $\mathcal{P}$ as $\mathcal{B}_{\mathcal{P}}$, or simply as $\mathcal{B}$. In practice, we are going to consider the dense sets $\mathbb{R}$ and $\mathbb{R}_{\geq 0}$ as time domains, 
or their rationals counterparts $\mathbb{Q}$ and $\mathbb{Q}_{>0}$. All the results of this paper stand regardless of which temporal domain, among $\mathbb{R}, \mathbb{R}_{\geq 0}, \mathbb{Q}, \mathbb{Q}_{\geq 0}$, is assumed ${ }^{2}$

The semantics of $\widetilde{M T L}$ formulas is given through a satisfaction relation $\models_{\mathrm{T}}$ : given a behavior $b \in \mathcal{B}$, an instant $t \in \mathbb{T}$ (sometimes called "current instant") and a MTL formula $\phi$, the satisfaction relation is defined inductively as follows.

$$
\begin{array}{lll}
b(t) \models_{\mathbb{T}} \mathrm{p} & \text { iff } & \mathrm{p} \in b(t) \\
b(t) \models_{\mathbb{T}} \widetilde{U}_{I}\left(\phi_{1}, \phi_{2}\right) & \text { iff } & \text { there exists } d \in I \text { such that } b(t+d) \models_{\mathrm{T}} \phi_{2} \\
& & \text { and, for all } u \in(0, d) \text { it is } b(t+u) \models_{\mathbb{T}} \phi_{1} \\
b(t) \models_{\mathbb{T}} \neg \phi & \text { iff } & b(t) \models_{\mathbb{T}} \phi \\
b(t) \models_{\mathbb{T}} \phi_{1} \wedge \phi_{2} & \text { iff } & b(t) \models_{\mathbb{T}} \phi_{1} \text { and } b(t) \models_{\mathbb{T}} \phi_{2}
\end{array}
$$

For clarity, let us also write out explicitly the derived semantics of the $\widetilde{\mathrm{R}}$ operator: $b(t) \models_{\mathbb{T}} \widetilde{\mathrm{R}}_{I}\left(\phi_{1}, \phi_{2}\right)$ iff for all $d \in I$, it is $b(t+d) \models_{\mathbb{R}} \phi_{2}$ or there exists a $u \in(0, d)$ such that $b(t+u) \models_{\mathbb{R}} \phi_{1}$.

From these definitions, we define initial satisfiability and global satisfiability as follows: a formula $\phi$ is initially satisfiable over a behavior $b$ iff $b(0) \models_{\mathbb{T}} \phi$; a formula $\phi$ is globally satisfiable over a behavior $b$ iff $\forall t \in \mathbb{T}: b(t) \models_{\mathbb{T}} \phi$, and we write $b \models_{\mathbb{T}} \phi$. The initial and global satisfiability relations allow one to identify a formula $\phi$ with the set of behaviors $\llbracket \phi \rrbracket_{T}$ that satisfy it according to each semantics; hence we introduce the notation $\llbracket \phi \rrbracket_{\mathrm{T}}^{0}=\left\{b \in \mathcal{B} \mid b(0) \models_{\mathrm{T}} \phi\right\}$ and $\llbracket \phi \rrbracket_{\mathbb{T}}=\left\{b \in \mathcal{B} \mid b \models_{\mathbb{T}} \phi\right\}$. Correspondingly, all formulas of some MTL variant identify a set of sets of behaviors which characterize the expressive power of that variant. We overload the notation and also denote by $\widetilde{\mathrm{MTL}}, \mathrm{MTL}, \widetilde{\mathrm{MTL}}^{\downarrow}$, and $\mathrm{MTL}^{\downarrow}$ the set of sets of behaviors identified by all strict non-matching, non-strict matching, strict matching, and non-strict non-matching formulas, respectively. It will be clear from the context whether we are referring to a set of formulas or to the corresponding set of sets of behaviors, and whether we are considering the initial or global satisfiability semantics.

Since the non-strict and matching variants have been defined in terms of $\widetilde{\text { MTL }}$ - and their definitions do not nest temporal operators - it is clear that the following relations hold: $\mathrm{MTL}^{\downarrow} \subseteq \mathrm{MTL} \subseteq \widetilde{\mathrm{MTL}}, \mathrm{MTL}^{\downarrow} \subseteq \widetilde{\mathrm{MTL}} \subseteq \widetilde{\mathrm{MTL}}$, $b \mathrm{MTL}^{\downarrow} \subseteq \mathrm{bMTL} \subseteq b \widetilde{\mathrm{MTL}}$, and $b \mathrm{MTL}^{\downarrow} \subseteq b \widetilde{\mathrm{MTL}} \subseteq$ ${ }^{\downarrow} \widetilde{\mathrm{MTL}}$.

\subsubsection{Non-Zenoness}

Behaviors over dense time are often subject to the non-Zenoness (also called finite variability [HR04, Rab03]) requirement [AL94, GM01]. A behavior $b \in \mathcal{B}$ is called non-Zeno if the truth value of any atomic proposition $\mathrm{p} \in \mathcal{P}$ changes in $b$ only finitely many times over any bounded interval of time. Formally, we require that for any $t \in \mathbb{T}$ there exists an $\epsilon>0$ such that, for all $\mathrm{p} \in \mathcal{P}$ : (1) either $\mathrm{p} \in b(u)$ for all $u \in(t, t+\epsilon)$ or $\mathrm{p} \notin b(u)$ for all $u \in(t, t+\epsilon)$; and (2) either $\mathrm{p} \in b(v)$ for all $v \in(t-\epsilon, t) \cap \mathbb{T}$ or $\mathrm{p} \notin b(v)$ for all $v \in(t-\epsilon, t) \cap \mathbb{T}$. In particular, it can be easily seen that $b \models_{\mathbb{R}} \square_{[0,+\infty)}(\widetilde{\bigcirc}(\mathrm{p}) \vee \widetilde{\bigcirc}(\neg \mathrm{p}))$ must hold for any non-Zeno behavior $b$ and atomic proposition $\mathrm{p}$.

This derived property can be "lifted" from atomic propositions to generic MTL formulas, by observing that MTL operators preserve non-Zenoness (the

\footnotetext{
${ }^{2}$ Even if we only consider future operator, bi-infinite time domains $\mathbb{R}$ and $\mathbb{Q}$ are introduced as they go "more naturally" with the global satisfiability semantics defined below.
} 
proof is straightforward by structural induction). Then, for any formula $\phi$ and non-Zeno behavior $b$ :

$$
b \models_{\mathbb{T}} \square_{[0,+\infty)}(\widetilde{\bigcirc}(\phi) \vee \widetilde{\bigcirc}(\neg \phi))
$$

Unless otherwise specified, in this paper we assume non-Zeno behaviors, with the exception of Section 5 where the impact of allowing non-Zeno behaviors will be analyzed. With non-Zeno behaviors, the following equivalence, which we proved in [FR07a], shows that the strict $\widetilde{\bigcirc}$ operator can be expressed with non-strict $\bigcirc$ operator over non-Zeno behaviors.

$$
\widetilde{\bigcirc}(\phi) \equiv \bigcirc(\phi) \vee(\neg \phi \wedge \neg \bigcirc(\neg \phi))
$$

\section{$3 \quad$ Nesting MTL over non-Zeno Behaviors}

This section shows that the four MTL variants: $\widetilde{\mathrm{MTL}}, \mathrm{MTL}, \widetilde{\mathrm{MTL}}{ }^{\downarrow}$, and MTL ${ }^{\downarrow}$ all have the same expressive power over non-Zeno behaviors, for both the initial and global satisfiability semantics. In fact, we provide a set of equivalences according to which one can replace each occurrence of strict until in terms of non-strict until, and each occurrence of non-matching until in terms of matching until. This shows that MTL $=\widetilde{\mathrm{MTL}}=\widetilde{\mathrm{MTL}}{ }^{\downarrow}=\mathrm{MTL}^{\downarrow}$. Note that the result holds regardless of whether the global or initial satisfiability relation in considered.

\subsection{Non-Strict as Expressive as Strict}

In [FR07a] we have shown that MTL $=\widetilde{\mathrm{MTL}}$; more precisely, the following equivalences have been proved, for $a>0$ (and $b>0$ in (6)).

$$
\begin{gathered}
\widetilde{\mathrm{U}}_{(a, b\rangle}\left(\phi_{1}, \phi_{2}\right) \equiv \diamond_{(a, b\rangle}\left(\phi_{2}\right) \wedge \square_{(0, a]}\left(\mathrm{U}_{(0,+\infty)}\left(\phi_{1}, \phi_{2}\right)\right) \\
\widetilde{\mathrm{U}}_{[a, b\rangle}\left(\phi_{1}, \phi_{2}\right) \equiv \widetilde{\mathrm{U}}_{(a, b\rangle}\left(\phi_{1}, \phi_{2}\right) \vee\left(\square_{(0, a)}\left(\phi_{1}\right) \wedge \diamond_{=a}\left(\phi_{2}\right)\right) \\
\widetilde{\mathrm{U}}_{(0, b\rangle}\left(\phi_{1}, \phi_{2}\right) \equiv \diamond_{(0, b\rangle}\left(\phi_{2}\right) \wedge \widetilde{\bigcirc}\left(\mathrm{U}_{(0,+\infty)}\left(\phi_{1}, \phi_{2}\right)\right) \\
\widetilde{\mathrm{U}}_{[0, b\rangle}\left(\phi_{1}, \phi_{2}\right) \equiv \widetilde{\mathrm{U}}_{(0, b\rangle}\left(\phi_{1}, \phi_{2}\right) \vee \phi_{2} \\
\widetilde{\mathrm{U}}_{[0,0]}\left(\phi_{1}, \phi_{2}\right) \equiv \phi_{2}
\end{gathered}
$$

Note that the $\diamond$ operator (and correspondingly the $\square$ operator as well) can be equivalently expressed with any of the until variants introduced beforehand, i.e., $\diamond_{I}(\phi) \equiv \widetilde{\cup}_{I}(\top, \phi) \equiv \cup_{I}(\top, \phi) \equiv \widetilde{U}_{I}^{\downarrow}(\top, \phi) \equiv \bigcup_{I}^{\downarrow}(\top, \phi)$. In particular, (3-7) provide a means to replace each occurrence of strict until with non-strict untils only. Now notice the following: if we replace each occurrence of formula $\phi_{2}$ in (3-7) with $\phi_{2} \wedge \phi_{1}$ - except for (6) which requires a slightly different treatment, which is however routine — we also have a proof that $\widetilde{\mathrm{MTL}}^{\downarrow}=\mathrm{MTL}^{\downarrow}$, according to the definition of the matching variants of the until operators. 


\subsection{Matching as Expressive as Non-Matching}

This section provides a set of equivalences to replace each occurrence of a strict non-matching operator with a formula that contains only strict matching operators; this shows that $\widetilde{\mathrm{MTL}}=\widetilde{\mathrm{MTL}}{ }^{\downarrow}$. To this end, let us first prove the following equivalence.

$$
\begin{aligned}
\widetilde{\mathrm{U}}_{(0, b\rangle}\left(\phi_{1}, \phi_{2}\right) \equiv & \widetilde{\mathrm{U}}_{(0, b\rangle}^{\downarrow}\left(\phi_{1}, \phi_{2}\right) \\
& \vee\left(\diamond_{(0, b\rangle}\left(\phi_{2}\right) \wedge \widetilde{\bigcirc}\left(\phi_{1}\right) \wedge \widetilde{\mathrm{R}}_{(0, b\rangle}^{\downarrow}\left(\phi_{2}, \bigcirc\left(\phi_{1}\right)\right)\right.
\end{aligned}
$$

Proof of Formula 8. Let us start with the $\Rightarrow$ direction, and let $t$ be the current instant. Assume that there exists a $d \in(0, b\rangle$ such that $b(t+d) \models_{\mathbb{R}} \phi_{2}$ and for all $u \in(t, t+d)$ it is $b(u) \models_{\mathbb{R}} \phi_{1}$. If also $b(t+d) \models_{\mathbb{R}} \phi_{1}$ or $b(t) \models_{\mathbb{R}} \widetilde{\bigcirc}\left(\phi_{2}\right)$ then $b(t) \models_{\mathbb{R}} \widetilde{\mathrm{U}}_{(0, b\rangle}^{\downarrow}\left(\phi_{1}, \phi_{2}\right)$, so assume that $b(t+d) \models_{\mathbb{R}} \neg \phi_{1}$ and $b(t) \models_{\mathbb{R}}$ $\widetilde{\bigcirc}\left(\neg \phi_{2}\right)$. Therefore, it is well-defined $d^{\prime}$, the smallest instant in $(t, t+b\rangle$ such that $b\left(d^{\prime}\right) \models_{\mathbb{R}} \phi_{2} \vee \widetilde{\bigcirc}\left(\phi_{2}\right)$; note that $d^{\prime} \leq t+d$. Note that clearly $b(t) \models_{\mathbb{R}} \widetilde{\bigcirc}\left(\phi_{1}\right)$ and $b(t) \models_{\mathbb{R}} \diamond_{(0, b\rangle}\left(\phi_{2}\right)$.

Let us first consider the case $b\left(d^{\prime}\right) \models_{\mathbb{R}} \phi_{2}$, and let $v \in(t, t+b\rangle$. If $v \geq t+d$, there exists a $w=t+d$ such that $w \in(t, v]$ and $b(w) \models_{\mathbb{R}} \phi_{2}$. If $v<t+d$ instead, then $v \in(t, t+d)$ so $b(v) \models_{\mathbb{R}} \bigcirc\left(\phi_{1}\right)$. All this shows that $b(t) \models_{\mathbb{R}}$ $\widetilde{\mathrm{R}}_{(0, b\rangle}^{\downarrow}\left(\phi_{2}, \bigcirc\left(\phi_{1}\right)\right)$, which completes the proof in this case.

Let us now consider the case $b\left(d^{\prime}\right) \models_{\mathbb{R}} \neg \phi_{2} \wedge \widetilde{\bigcirc}\left(\phi_{2}\right)$; hence $d^{\prime}<t+d$. So, let us assume that $\phi_{2}$ holds over the interval $\left(d^{\prime}, d^{\prime}+\epsilon\right)$ for some $\epsilon>0$. Now, let $v \in(t, t+b\rangle$. If $v>d^{\prime}$, there exists a $w=d^{\prime}+\min \left(v-d^{\prime}, \epsilon\right) / 2$ such that $w \in(t, v]$ and $b(w) \models_{\mathbb{R}} \phi_{2}$. If $v \leq d^{\prime}$ instead, then $v \in\left(t, d^{\prime}\right] \subset(t, t+d)$ so $b(v) \models_{\mathbb{R}} \bigcirc\left(\phi_{1}\right)$, as $\phi_{1}$ holds throughout $(t, t+d)$. All this shows that $b(t) \models_{\mathbb{R}} \widetilde{R}_{(0, b\rangle}^{\downarrow}\left(\phi_{2}, \bigcirc\left(\phi_{1}\right)\right)$, which completes the proof in this case as well.

Let us now consider the $\Leftarrow$ direction, and let $t$ be the current instant. If $b(t) \models_{\mathbb{R}} \widetilde{U}_{(0, b\rangle}^{\downarrow}\left(\phi_{1}, \phi_{2}\right)$ clearly also $b(t) \models_{\mathbb{R}} \widetilde{U}_{(0, b\rangle}\left(\phi_{1}, \phi_{2}\right)$ a fortiori. So let us assume that $\widetilde{\mathbf{U}}_{(0, b\rangle}^{\downarrow}\left(\phi_{1}, \phi_{2}\right)$ is false at $t$; note that this subsumes that $b(t) \models_{\mathbb{R}}$ $\widetilde{\bigcirc}\left(\phi_{2} \wedge \phi_{1}\right)$.

Let us remark that we can assume that $\widetilde{\bigcirc}\left(\neg \phi_{2}\right)$ holds at $t$, because $\widetilde{\bigcirc}\left(\phi_{1}\right)$ and $\neg \widetilde{\bigcirc}\left(\phi_{2} \wedge \phi_{1}\right)$ both hold. Therefore, it is well-defined $u$, the smallest instant in $(t, t+b\rangle$ such that $b(u) \models_{\mathbb{R}} \phi_{2} \vee \widetilde{\bigcirc}\left(\phi_{2}\right)$. Note that this implies that $\phi_{2}$ is false throughout $(t, u\rangle$, with the interval right-open iff $\phi_{2}$ holds at $u$.

Let us first consider the case $b(u) \models_{\mathbb{R}} \phi_{2}$. Let $v$ be a generic instant in $(t, u)$; recall that $\phi_{2}$ is false throughout $(t, u) \supset(t, v]$. Therefore it must be $b(v) \models_{\mathbb{R}}$ $\bigcirc\left(\phi_{1}\right)$ for $b(t) \models_{\mathbb{R}} \widetilde{R}_{(0, b\rangle}^{\downarrow}\left(\phi_{2}, \bigcirc\left(\phi_{1}\right)\right)$ to be true. So, $\phi_{1}$ holds throughout $(t, u)$ and $\phi_{2}$ holds at $u$, which means that $b(t) \models_{\mathbb{R}} \widetilde{U}_{(0, b\rangle}\left(\phi_{1}, \phi_{2}\right)$.

Let us now consider the other case $b(u) \models_{\mathbb{R}} \neg \phi_{2} \wedge \widetilde{\bigcirc}\left(\phi_{2}\right)$. Let $v$ be a generic instant in $(t, u]$; recall that $\phi_{2}$ is false throughout $(t, u] \supseteq(t, v]$. From $b(t) \models_{\mathbb{R}}$ $\widetilde{\mathrm{R}}_{(0, b\rangle}^{\downarrow}\left(\phi_{2}, \bigcirc\left(\phi_{1}\right)\right)$ it must be $b(v) \models_{\mathbb{R}} \bigcirc\left(\phi_{1}\right)$. Overall, $\phi_{1}$ holds throughout $(t, u+\epsilon]$ for some $\epsilon>0$, as in particular $b(t+u) \models_{\mathbb{R}} \bigcirc\left(\phi_{1}\right)$. Clearly, this subsumes $b(t) \models_{\mathbb{R}} \widetilde{U}_{(0, b\rangle}\left(\phi_{1}, \phi_{2}\right)$. 
The case for $a>0$ can be handled simply by relying on the previous equivalence. In fact, the following equivalence is easily seen to hold $\sqrt{3}^{3}$

$$
\begin{aligned}
\widetilde{\mathrm{U}}_{(a, b\rangle}\left(\phi_{1}, \phi_{2}\right) \equiv & \widetilde{\mathrm{U}}_{(a, b\rangle}^{\downarrow}\left(\phi_{1}, \phi_{2}\right) \\
& \vee\left(\square_{(0, a]}\left(\phi_{1}\right) \wedge \vartheta_{=a}\left(\widetilde{\mathrm{U}}_{(0, b-a\rangle}\left(\phi_{1}, \phi_{2}\right)\right)\right)
\end{aligned}
$$

The cases for left-closed intervals are also derivable straightforwardly as:

$$
\widetilde{\mathrm{U}}_{[0, b\rangle}\left(\phi_{1}, \phi_{2}\right) \equiv \phi_{2} \vee \widetilde{\mathrm{U}}_{(0, b\rangle}\left(\phi_{1}, \phi_{2}\right)
$$

and

$$
\widetilde{\mathrm{U}}_{[a, b\rangle}\left(\phi_{1}, \phi_{2}\right) \equiv\left(\diamond_{=a}\left(\phi_{2}\right) \wedge \square_{(0, a)}\left(\phi_{1}\right)\right) \vee \widetilde{\mathrm{U}}_{(a, b\rangle}\left(\phi_{1}, \phi_{2}\right)
$$

Finally, let us note that the $\bigcirc(\phi)$ operator can be expressed equivalently with strict matching operators as $\phi \wedge \widetilde{\mathrm{U}}_{(0,+\infty)}^{\downarrow}(\phi, \top)$. In fact, $\bigcirc(\phi)$ means that $\phi$ holds over an interval $[x, x+\epsilon)$ for some $\epsilon>0$; therefore, $\phi$ also holds over a closed interval such as $[x, x+\epsilon / 2]$, as required by $\phi \wedge \widetilde{\mathrm{U}}_{(0,+\infty)}^{\downarrow}(\phi, \top)$, and vice versa.

All in all (8-11) provide a means to replace every occurrence of strict nonmatching until with a formula that contains only strict matching untils. This shows that $\widetilde{\mathrm{MTL}}=\widetilde{\mathrm{MTL}}{ }^{\downarrow}$, completing our set of equivalences for non-Zeno behaviors.

\section{Flat MTL}

Section 3 has shown the equivalence of all (non-)strict and (non-)matching MTL variants for non-Zeno behaviors. It is apparent, however, that the equivalences between the various until variants introduce nesting of temporal operators, that is they change flat formulas into nesting ones. This section shows that this is inevitable, as the relative expressiveness relations change if we consider flat formulas only. More precisely, we prove that both non-strictness and matchingness lessen the expressive power of MTL flat formulas, so that the strict non-matching variant is shown to be the most expressive. We also show that, as one would expect, even this most expressive flat variant is less expressive than any nesting variant. All separation results are proved under both the initial satisfiability and the global satisfiability semantics.

\subsection{Non-Strict Less Expressive Than Strict}

This section shows that $b$ MTL $\subset$ b $\widetilde{M T L}$; let us outline the technique used to prove this fact. We provide a strict flat formula $\alpha \in b \widetilde{\mathrm{MTL}}$ and we prove that it has no equivalent non-strict flat formula. The proof goes adversarially: assume $\beta \in$ bMTL is a non-strict flat formula equivalent to $\alpha$, and let $\rho$ be the granularity of $\beta$. From $\rho$ we build two behaviors $b_{\top}^{\rho}$ and $b_{\perp}^{\rho}$ such that any bMTL

\footnotetext{
${ }^{3}$ Although (9) and (11) introduce punctual intervals, it is possible to avoid the use of such intervals, following an approach similar to that in [FR07a] for the translation of strict operators to strict ones.
} 


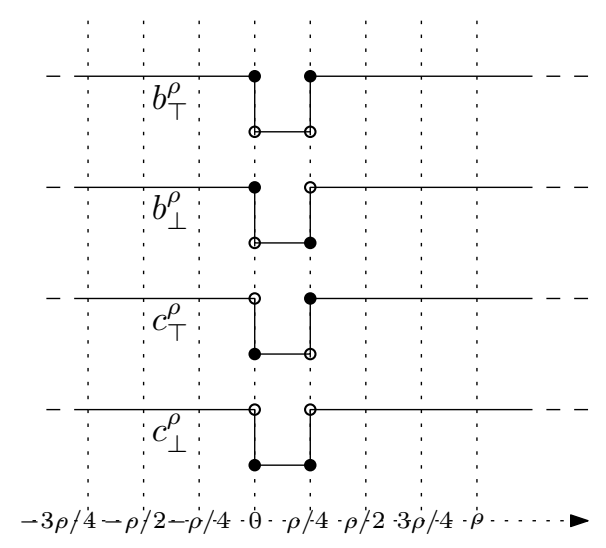

Figure 1: The behaviors $b_{\top}^{\rho}, b_{\perp}^{\rho}, c_{\top}^{\rho}, c_{\perp}^{\rho}$.

formula of granularity $\rho$ (and $\beta$ in particular) cannot distinguish between them, i.e. it is either satisfied by both or by none. On the contrary, $\alpha$ is satisfied by $b_{\top}^{\rho}$ but not by $b_{\perp}^{\rho}$, for all $\rho$. This shows that no equivalent non-strict flat formula can exist, and thus b $\widetilde{\mathrm{MTL}} \nsubseteq b \mathrm{MTL}$. From $b \mathrm{MTL} \subseteq b \widetilde{\mathrm{MTL}}$ we conclude that $b \mathrm{MTL} \subset$ b $\widehat{\mathrm{MTL}}$.

As in all separation results, the details of the proofs are rather involved; this is even more the case when considering the global satisfiability semantics; throughout we will try to provide some intuition leaving out the lowest-level details that are straightforward.

Let us define the following families of behaviors over $\{\mathrm{p}\}$. For any given $\rho>0$, let $b_{\top}^{\rho}$ and $b_{\perp}^{\rho}$ be defined as follows: $\mathrm{p} \in b_{\top}^{\rho}(t)$ iff $t \leq 0$ or $t \geq \rho / 4$; and $\mathrm{p} \in b_{\perp}^{\rho}(t)$ iff $t \leq 0$ or $t>\rho / 4$. Similarly, for any given $\rho>0, c_{\top}^{\rho}$ and $c_{\perp}^{\rho}$ are defined as follows: $\mathrm{p} \in c_{\top}^{\rho}(t)$ iff $\mathrm{p} \in b_{\top}^{\rho}(t)$ and $t \neq 0$; and $\mathrm{p} \in c_{\perp}^{\rho}(t)$ iff $\mathrm{p} \in \bar{b}_{\perp}^{\rho}(t)$ and $t \neq 0$. Note that $b_{\top}^{\rho}(t)=b_{\perp}^{\rho}(t)$ and $c_{\top}^{\rho}(t)=c_{\perp}^{\rho}(t)$ for all $t \neq \rho / 4$, and that $b_{\top}^{\rho}(t)=c_{\top}^{\rho}(t)$ and $b_{\perp}^{\rho}(t)=c_{\perp}^{\rho}(t)$ for all $t \neq 0$. Let us also define the sets of behaviors $\mathcal{B}_{\top}=\bigcup_{\rho \in \mathbb{R}_{>0}} b_{\top}^{\rho}$ and $\mathcal{B}_{\perp}=\bigcup_{\rho \in \mathbb{R}_{>0}} b_{\perp}^{\rho}$. The behaviors $b_{\top}^{\rho}, b_{\perp}^{\rho}, c_{\top}^{\rho}, c_{\perp}^{\rho}$ are pictured in Figure 1 .

\subsubsection{Initial Satisfiability}

Let us first assume the initial satisfiability semantics; we show that no bMTL formula $\phi$ with granularity $\rho$ distinguishes initially between $b_{\top}^{\rho}$ and $b_{\perp}^{\rho}$. To this end, we prove the following.

Lemma 1. For any bMTL formula $\phi$ of granularity $\rho$, it is $b_{\top}^{\rho}(0) \models_{\mathbb{R}} \phi$ iff $b_{\perp}^{\rho}(0) \models_{\mathrm{R}} \phi$.

Proof. We prove the lemma by induction on the structure of $\phi$.

- $\phi=\beta$, where $\beta$ is a Boolean combination of $\mathrm{p}$ and $\neg \mathrm{p}$. Since $b_{T}^{\rho}(0)=$ $b_{\perp}^{\rho}(0)$ this case follows trivially. Also note that $\beta$ is equivalent to one of $\mathrm{p}, \neg \mathrm{p}, \top, \perp$ over both $b_{\top}^{\rho}$ and $b_{\perp}^{\rho}$.

- $\phi=\neg \phi^{\prime}$. By inductive hypothesis it is $b_{\top}^{\rho}(0) \models_{\mathbb{R}} \phi^{\prime}$ iff $b_{\perp}^{\rho}(0) \models_{\mathbb{R}} \phi^{\prime}$, so clearly $b_{\top}^{\rho}(0) \models_{\mathbb{R}} \neg \phi^{\prime}$ iff $b_{\perp}^{\rho}(0) \models_{\mathbb{R}} \neg \phi^{\prime}$. 
- $\phi=\phi_{1} \wedge \phi_{2}$. By inductive hypothesis, $b_{\top}^{\rho}(0) \models_{\mathbb{R}} \phi_{1}$ iff $b_{\perp}^{\rho}(0) \models_{\mathbb{R}} \phi_{1}$, and $b_{\top}^{\rho}(0) \models_{\mathbb{R}} \phi_{2}$ iff $b_{\perp}^{\rho}(0) \models_{\mathbb{R}} \phi_{2}$. Thus $b_{\top}^{\rho}(0) \models_{\mathbb{R}} \phi_{1} \wedge \phi_{2}$ iff $b_{\perp}^{\rho}(0) \models_{\mathbb{R}} \phi_{1} \wedge \phi_{2}$.

- $\phi=\mathrm{U}_{I}\left(\beta_{1}, \beta_{2}\right)$, with $I=\langle l, u\rangle$ and: (1) $l=k_{1} \rho$ for some $k_{1} \in \mathbb{N}$; and (2) $u=k_{2} \rho$ or $u=+\infty$, for some $k_{1} \leq k_{2} \in \mathbb{N}$. Note that we can assume $0 \notin I$ without loss of generality, as $\mathrm{U}_{[0, u\rangle}\left(\beta_{1}, \beta_{2}\right) \equiv \beta_{2} \vee \mathrm{U}_{(0, u\rangle}\left(\beta_{1}, \beta_{2}\right)$. We also assume that $I$ is non-empty; this is also without loss of generality.

We consider several cases for $\beta_{1}, \beta_{2}$.

- If $\beta_{1} \equiv \beta_{2} \equiv \top$ then clearly $b_{\top}^{\rho}(0) \models_{\mathbb{R}} \phi$ and $b_{\perp}^{\rho}(0) \models_{\mathbb{R}} \phi$.

- If $\beta_{2} \equiv \perp$ then clearly $b_{\top}^{\rho}(0) \not \nvdash_{\mathbb{R}} \phi$ and $b_{\perp}^{\rho}(0) \not \nvdash_{\mathbb{R}} \phi$.

- If $\beta_{1} \equiv \perp$ then $b_{\top}^{\rho}(0) \nvdash_{\mathbb{R}} \phi$ and $b_{\perp}^{\rho}(0) \nvdash_{\mathbb{R}} \phi$, as $0 \notin I$.

- If $\beta_{1} \equiv \top$ and $\beta_{2}$ is one of $\mathrm{p}$ or $\neg \mathrm{p}$, we have $\phi \equiv \diamond_{I}\left(\beta_{2}\right)$.

If $l=0$, then $u>l$, which entails $u \geq \rho$. Any interval of the form $\langle 0, \rho\rangle$ encompasses both instants where $\mathrm{p}$ holds and instants where $\neg p$ holds. Thus, $b_{+}^{\rho}(0) \models_{\mathbb{R}} \phi$ and $b_{\perp}^{\rho}(0) \models_{\mathbb{R}} \phi$ in this case.

If $l>0$ then $l \geq \rho$. Then, whatever $u \geq l$ is, it is clear that $\mathrm{p}$ holds throughout the non-empty interval $\langle l, u\rangle$. Therefore, if $\beta_{2} \equiv \mathrm{p}$ we have $b_{\top}^{\rho}(0) \models_{\mathbb{R}} \phi$ and $b_{\perp}^{\rho}(0) \models_{\mathbb{R}} \phi$; otherwise $\beta_{2} \equiv \neg \mathrm{p}$, and $b_{\top}^{\rho}(0) \not \forall_{\mathbb{R}} \phi$ and $b_{\perp}^{\rho}(0) \nvdash_{\mathbb{R}} \phi$.

- If $\beta_{1} \equiv \mathrm{p}$ or $\beta_{1} \equiv \neg \mathrm{p}$, then $\phi$ does not hold unless $\bigcirc\left(\beta_{1}\right)$ holds (still because $0 \notin I$ ). Since the value of $\mathrm{p}$ changes from 0 to its immediate future, it is $b_{\top}^{\rho}(0) \not \nvdash_{\mathbb{R}} \phi$ and $b_{\perp}^{\rho}(0) \not \nvdash_{\mathbb{R}} \phi$.

Lemma 1 leads straightforwardly to the desired separation result.

Theorem 2. Under the initial satisfiability semantics, bMTL $\subset$ b $\widetilde{\mathrm{MTL}}$.

Proof. Let us show that the b $\widetilde{\mathrm{MTL}}$ formula $\Sigma=\widetilde{\mathrm{U}}_{(0,+\infty)}(\neg \mathrm{p}, \mathrm{p})$ has no equivalent bMTL formula. $\Sigma$ can distinguish initially between the families of behaviors $\mathcal{B}_{\top}, \mathcal{B}_{\perp}$, as for any $b \in \mathcal{B}_{\top}, b^{\prime} \in \mathcal{B}_{\perp}$, it is $b(0) \models_{\mathbb{R}} \Sigma$ and $b^{\prime}(0) \nvdash_{\mathbb{R}} \Sigma$. Let us assume that $\sigma$ is an bMTL formula of granularity $\rho$ equivalent to $\Sigma$. However, from Lemma 1 it follows that $b_{\top}^{\rho}(0) \models_{\mathbb{R}} \sigma$ iff $b_{\perp}^{\rho}(0) \models_{\mathbb{R}} \sigma$. Therefore, $\sigma$ is not equivalent to $\Sigma$.

\subsubsection{Global Satisfiability}

Let us now prove an analogous of Theorem 2 for the global satisfiability semantics.

Consequences of Lemma 1 for Global Satisfiability. First of all, let us recall that, in the global satisfiability semantics, $b \models_{\mathbb{T}} \phi$ means that for all $t \in \mathbb{T}$ it is $b(t) \models_{\mathbb{T}} \phi$, whereas $b \nvdash_{\mathbb{T}} \phi$ means that there exists a $t \in \mathbb{T}$ such that $b(t) \nvdash_{\mathbb{T}} \phi$. Note that there is an duality between the definitions of satisfiability and unsatisfiability.

For the sake of argument, let us pretend that b $\widetilde{\mathrm{MTL}}$ and bMTL have equivalent expressiveness and see what the consequences of this fact would be. This means that there exists a mapping $\tau$ from formulas in b $\widetilde{\mathrm{MTL}}$ to formulas in bMTL such that, for any b $\widetilde{\mathrm{MTL}}$ formula $\phi$ and any behavior $b$ it is $b \models_{\mathbb{T}} \phi$ iff 
$b \models_{\mathbb{T}} \tau(\phi)$. However, as a consequence of Lemma 1, any such translation $\tau$ must be non-local and non-homomorphic or non-idempotent.

Non-local means that $\tau$ cannot be such that $b(t) \models_{\mathbb{T}} \phi$ iff $b(t) \models_{\mathbb{T}} \tau(\phi)$ for all $t \in \mathbb{T}$, and any behavior $b$. Otherwise, let $\Sigma$ be the b $\widetilde{\mathrm{MTL}}$ formula as in the proof of Theorem 2. Then, the bMTL formula $\tau(\Sigma)$ would be such that, for any $b_{\top}^{\rho} \in \mathcal{B}_{\top}, b_{\perp}^{\rho} \in \mathcal{B}_{\perp}, b_{\top}^{\rho}(0) \models_{\mathbb{T}} \tau(\Sigma)$ and $b_{\perp}^{\rho}(0) \nvdash_{\mathbb{T}} \tau(\Sigma)$, which contradicts Lemma 1 .

Non-homomorphic or non-idempotent means that $\tau$ cannot be such that $\tau\left(\phi_{1} \vee \phi_{2}\right)=\tau\left(\phi_{1}\right) \vee \tau\left(\phi_{2}\right)$ and $\tau(\tau(\phi))=\tau(\phi)$; in other words it is not an homomorphism with respect to disjunction or it is not an idempotence. In fact, let $\Omega$ be the b $\widetilde{\mathrm{MTL}}$ formula $\Sigma \vee \bigcirc(\neg p) \vee \widetilde{\bigcirc}(\mathrm{p})$, and let $\rho$ be the granularity of $\tau(\Omega)$. Then one can check that $b_{\top}^{\rho} \models_{\mathbb{T}} \Omega$ and $b_{\perp}^{\rho} \forall_{\mathbb{T}} \Omega$, so it must be $b_{\mathrm{T}}^{\rho} \models_{\mathbb{T}} \tau(\Omega)$ and $b_{\perp}^{\rho} \forall_{\mathbb{T}} \tau(\Omega)$. Now, consider the $b \widetilde{\mathrm{MTL}}$ formula $\Omega \vee \tau(\Omega)$. Notice that $\Omega$ holds over $b_{\perp}^{\rho}$ for all $t \neq 0$, and recall that, from Lemma 1 , it must be $b_{\mathrm{T}}^{\rho}(0) \models_{\mathrm{T}} \tau(\Omega)$ and $b_{\perp}^{\rho}(0) \models_{\mathbb{T}} \tau(\Omega)$. Therefore, both $b_{\top}^{\rho} \models_{\mathbb{T}} \Omega \vee \tau(\Omega)$ and $b_{\perp}^{\rho} \models_{\mathbb{T}} \Omega \vee \tau(\Omega)$. If $\tau$ is compositional, however, it would be $\tau(\Omega \vee \tau(\Omega))=\tau(\Omega) \vee \tau(\Omega)=\tau(\Omega)$. Then, $b_{\top}^{\rho} \models_{\mathbb{T}} \tau(\Omega \vee \tau(\Omega))$ and $b_{\perp}^{\rho} \forall_{\mathbb{T}} \tau(\Omega \vee \tau(\Omega))$ so that the resulting non-strict formula is not equivalent to the strict one.

Since locality, idempotence and homorphicity are reasonable properties of a translation function $\tau$, the above reasoning suggests that $\tau$ does not exist. We are now going to prove this intuition.

Proofs for Global Satisfiability. Let us now prove that bMTL formulas cannot distinguish between the three families of behaviors $b_{\top}^{\rho}, b_{\perp}^{\rho}, c_{\perp}^{\rho}$ for all instants $t<0$.

Lemma 3. For any bMTL formula $\phi$ of granularity $\rho$ and any instant $t<0$, it is: $b_{\top}^{\rho}(t) \models_{\mathbb{R}} \phi$ iff $b_{\perp}^{\rho}(t) \models_{\mathbb{R}} \phi$, or $b_{\perp}^{\rho}(t) \models_{\mathbb{R}} \phi$ iff $c_{\perp}^{\rho}(t) \models_{\mathbb{R}} \phi$.

Proof. The proof is by induction on the structure of $\phi$; throughout, $t$ is any fixed instant less than 0 .

- $\phi=\beta$. Since $b_{\top}^{\rho}(t)=b_{\perp}^{\rho}(t)=c_{\perp}^{\rho}(t)$, this case follows trivially.

- $\phi=\neg \phi^{\prime}$. By inductive hypothesis, either $b_{\top}^{\rho}(t) \models_{\mathbb{R}} \phi^{\prime}$ iff $b_{\perp}^{\rho}(t) \models_{\mathbb{R}} \phi^{\prime}$, or $b_{\perp}^{\rho}(t) \models_{\mathbb{R}} \phi^{\prime}$ iff $c_{\perp}^{\rho}(t) \models_{\mathbb{R}} \phi^{\prime}$. In the former case, $b_{\top}^{\rho}(t) \models_{\mathbb{R}} \phi$ iff $b_{\perp}^{\rho}(t) \models_{\mathbb{R}} \phi$, otherwise $b_{\perp}^{\rho}(t) \models_{\mathbb{R}} \phi$ iff $c_{\perp}^{\rho}(t) \models_{\mathbb{R}} \phi$.

- $\phi=\mathrm{U}_{I}\left(\beta_{1}, \beta_{2}\right)$. We consider several cases for $\beta_{1}, \beta_{2}$.

- If $\beta_{1} \equiv \beta_{2} \equiv \mathrm{T}$, then $b_{\mathrm{T}}^{\rho}(t) \models_{\mathbb{R}} \phi, b_{\perp}^{\rho}(t) \models_{\mathbb{R}} \phi, c_{\perp}^{\rho}(t) \models_{\mathbb{R}} \phi$.

- If $\beta_{1} \equiv \perp$ or $\beta_{2} \equiv \perp$, then $b_{\top}^{\rho}(t) \not \nvdash_{\mathbb{R}} \phi, b_{\perp}^{\rho}(t) \not \nvdash_{\mathbb{R}} \phi, c_{\perp}^{\rho}(t) \nvdash_{\mathbb{R}} \phi$.

- If $\beta_{1} \equiv T$ and $\beta_{1}$ is one of $\mathrm{p}, \neg \mathrm{p}$, we have $\phi \equiv \diamond_{I}\left(\beta_{2}\right)$. If $l=u=k \rho$ and $t+k \rho \neq \rho / 4$, we have $b_{\top}^{\rho}(t) \models_{\mathbb{R}} \phi$ iff $b_{\perp}^{\rho}(t) \models_{\mathbb{R}} \phi$ iff $c_{\perp}^{\rho}(t) \models_{\mathbb{R}} \phi$. If $l=u=k \rho$ and $t+k \rho=\rho / 4$, we have $b_{\top}^{\bar{\rho}}(t) \not \models_{\mathbb{R}} \phi$ iff $b_{\perp}^{\bar{\rho}}(t) \models_{\mathbb{R}} \phi$ iff $c_{\perp}^{\rho}(t) \models_{\mathbb{R}} \phi$. If $l<u$ (and thus $u-l \geq \rho$ ) and $\beta_{2} \equiv \mathrm{p}$, then $b_{\top}^{\rho}(t) \models_{\mathbb{R}} \phi$ and $b_{\perp}^{\rho}(t) \models_{\mathbb{R}} \phi$ and $c_{\perp}^{\rho}(t) \models_{\mathbb{R}} \phi$, as any interval of size $\rho$ contains instants where $\mathrm{p}$ holds. If $l<u$ and $\beta_{2} \equiv \neg \mathrm{p}$, we have: (1) $(t+l>\rho / 4) \vee(t+u<0) \vee(t+l=\rho / 4 \wedge l \notin I) \vee(t+u=0 \wedge u \notin I)$ and $b_{\top}^{\rho}(t) \nvdash_{\mathbb{R}} \phi$ and $b_{\perp}^{\rho}(t) \nvdash_{\mathbb{R}} \phi$ and $c_{\perp}^{\rho}(t) \nvdash_{\mathbb{R}} \phi$; or $(2) t+l=\rho / 4$ and $l \in I$ and $b_{\top}^{\rho}(t) \nvdash_{\mathbb{R}} \phi$ and $b_{\perp}^{\rho}(t) \models_{\mathbb{R}} \phi$ and $c_{\perp}^{\rho}(t) \models_{\mathbb{R}} \phi$; or $(3)$ 
$t+u=0$ and $u \in I$ and $b_{\top}^{\rho}(t) \nvdash_{\mathbb{R}} \phi$ and $b_{\perp}^{\rho}(t) \nvdash_{\mathbb{R}} \phi$ and $c_{\perp}^{\rho}(t) \models_{\mathbb{R}} \phi$; or (4) $t+l<\rho / 4$ and $t+u>0$ and $b_{\top}^{\rho}(t) \models_{\mathbb{R}} \phi$ and $b_{\perp}^{\rho}(t) \models_{\mathbb{R}} \phi$ and $c_{\perp}^{\rho}(t) \models_{\mathbb{R}} \phi$.

- If $\beta_{1} \equiv \beta_{2} \equiv \mathrm{p}$ and $l=0$, then $\phi$ holds iff $\bigcirc(\mathrm{p})$, so $b_{\top}^{\rho}(t) \models_{\mathbb{R}} \phi$ and $b_{\perp}^{\rho}(t) \models_{\mathbb{R}} \phi$ and $c_{\perp}^{\rho}(t) \models_{\mathbb{R}} \phi$. If instead $l>0$ and $(t+l<0)$ then also $b_{\top}^{\rho}(t) \models_{\mathbb{R}} \phi$ and $b_{\perp}^{\rho}(t) \models_{\mathbb{R}} \phi$ and $c_{\perp}^{\rho}(t) \models_{\mathbb{R}} \phi$. If $l>0$ but $t+l>0$ or $t+l=0 \wedge l \notin I$ then $b_{\top}^{\rho}(t) \forall_{\mathbb{R}} \phi$ and $b_{\perp}^{\rho}(t) \forall_{\mathbb{R}} \phi$ and $c_{\perp}^{\rho}(t) \forall_{\mathbb{R}} \phi$. Finally, if $l>0, t+l=0 \wedge l \in I$ then $b_{\top}^{\bar{\rho}}(t) \models_{\mathbb{R}} \phi$ and $b_{\perp}^{\rho}(t) \models_{\mathbb{R}} \phi$ and $c_{\perp}^{\rho}(t) \not \nvdash_{\mathbb{R}} \phi$.

- If $\beta_{1} \equiv \neg \mathrm{p}, \phi$ holds only if $\bigcirc(\neg \mathrm{p})$ holds; therefore $b_{\mathrm{T}}^{\rho}(t) \not \nvdash_{\mathbb{R}} \phi$ and $b_{\perp}^{\rho}(t) \nvdash_{\mathbb{R}} \phi$ and $c_{\perp}^{\rho}(t) \nvdash_{\mathbb{R}} \phi$.

- If $\beta_{1} \equiv \mathrm{p}$ and $\beta_{2} \equiv \neg \mathrm{p}$ then surely $b_{\top}^{\rho}(t) \nvdash_{\mathbb{R}} \phi$ and $b_{\perp}^{\rho}(t) \nvdash_{\mathbb{R}} \phi$ and $c_{\perp}^{\rho}(t) \not \nvdash_{\mathbb{R}} \phi$.

Overall, notice that it is $b_{\top}^{\rho} \models_{\mathbb{R}} \phi$ iff $b_{\perp}^{\rho} \models_{\mathbb{R}} \phi$ iff $c_{\perp}^{\rho} \models_{\mathbb{R}} \phi$, unless: (a) $b_{\top}^{\rho} \not \models_{\mathbb{R}} \phi$ iff $b_{\perp}^{\rho} \models_{\mathbb{R}} \phi$ iff $c_{\perp}^{\rho} \models_{\mathbb{R}} \phi$ and $t+k \rho=\rho / 4$ or $t+k \rho=0$ for some positive integer $k$; or (b) $b_{\top}^{\rho} \models_{\mathbb{R}} \phi$ iff $b_{\perp}^{\rho} \models_{\mathbb{R}} \phi$ iff $c_{\perp}^{\rho} \forall_{\mathbb{R}} \phi$ and $t+h \rho=0$ for some positive integer $h$.

- $\phi=\phi_{1} \wedge \phi_{2}$. If $b_{\top}^{\rho}(t) \models_{\mathbb{R}} \phi_{i}$ iff $b_{\perp}^{\rho}(t) \models_{\mathbb{R}} \phi_{i}$ iff $c_{\perp}^{\rho}(t) \models_{\mathbb{R}} \phi_{i}$ for both $i=1,2$, then clearly $b_{\top}^{\rho}(t) \models_{\mathbb{R}} \phi$ iff $b_{\perp}^{\rho}(t) \models_{\mathbb{R}} \phi$ iff $c_{\perp}^{\rho}(t) \models_{\mathbb{R}} \phi$ and we are done.

Otherwise, assume that $b_{\top}^{\rho}(t) \models_{\mathbb{R}} \phi_{i}$ iff $b_{\perp}^{\rho}(t) \models_{\mathbb{R}} \phi_{i}$ iff $c_{\perp}^{\rho}(t) \models_{\mathbb{R}} \phi_{i}$ for some $i$, and $b_{\top}^{\rho}(t) \nvdash_{\mathbb{R}} \phi_{j}$ iff $b_{\perp}^{\rho}(t) \models_{\mathbb{R}} \phi_{j}$ iff $c_{\perp}^{\rho}(t) \models_{\mathbb{R}} \phi_{j}$ and $t+k \rho=\rho / 4$ for $j \neq i$. In this case we have $b_{\top}^{\rho}(t) \models_{\mathbb{R}} \phi$ iff $b^{\rho}(t) \models_{\mathbb{R}} \phi$ iff $c^{\rho}(t) \models_{\mathbb{R}} \phi$ and $t+k \rho=\rho / 4$. Similarly, if $b_{\top}^{\rho}(t) \models_{\mathbb{R}} \phi_{i}$ iff $b_{\perp}^{\rho_{\perp}}(t) \models_{\mathbb{R}} \phi_{i}$ iff $c_{\perp}^{\rho}(t) \models_{\mathbb{R}} \phi_{i}$ for some $i$, and $b_{\top}^{\rho}(t) \models_{\mathbb{R}} \phi_{j}$ iff $b_{\perp}^{\rho}(t) \models_{\mathbb{R}} \phi_{j}$ iff $c_{\perp}^{\rho_{\perp}}(t) \forall_{\mathbb{R}} \phi_{j}$ and $t+h \rho=0$ for $j \neq i$, we have $b_{\top}^{\rho}(t) \models_{\mathbb{R}} \phi$ iff $b_{\perp}^{\rho}(t) \models_{\mathbb{R}} \phi$ iff $c_{\perp}^{\rho}(t) \nvdash_{\mathbb{R}} \phi$ and $t+h \rho=0$.

Finally, consider the case $b_{\top}^{\rho}(t) \nvdash_{\mathbb{R}} \phi_{i}$ iff $b_{\perp}^{\rho}(t) \models_{\mathbb{R}} \phi_{i}$ iff $c_{\perp}^{\rho}(t) \models_{\mathbb{R}} \phi_{i}$ and $t+k \rho=\rho / 4$ for some $i$, and $b_{\top}^{\rho}(t) \models_{\mathbb{R}} \phi_{j}$ iff $b_{\perp}^{\rho}(t) \models_{\mathbb{R}} \phi_{j}$ iff $c_{\perp}^{\rho}(t) \models_{\mathbb{R}} \phi_{j}$ and $t+h \rho=0$ for $j \neq i$. This case, however, is not possible as $t+k \rho=$ $\rho / 4=\rho / 4+t+h \rho$ implies $(k-h) \rho=\rho / 4$ which is impossible as $k$ and $h$ are integers. To give some intuition, this is due to the granularity: in other words, from the same $t$ we cannot reference both 0 and $\rho / 4$, since they are less than $\rho$ time instants apart. Finally also note that this restriction can be "lifted" to the conjunction itself, to go with the inductive hypothesis.

Through Lemma 3 we can extend Theorem 2 to the global satisfiability semantics.

Theorem 4. Under the global satisfiability semantics, bMTL $\subset$ b $\widetilde{\mathrm{MTL}}$.

Proof. Let us show that the b $\widetilde{\mathrm{MTL}}$ formula:

$$
\Omega=\widetilde{U}_{(0,+\infty)}(\neg p, p) \vee \bigcirc(\neg p) \vee \widetilde{O}(p)
$$

has no equivalent $b$ MTL formula. It is simple to check that, for all $b \in \mathcal{B}_{\top}, b^{\prime} \in$ $\mathcal{B}_{\perp}$ it is $b \models_{\mathbb{R}_{>0}} \Omega$ and $b^{\prime} \forall_{\mathbb{R}_{>0}} \Omega$; more precisely, it is $b^{\prime}(0) \forall_{\mathbb{R}} \Omega$ and, for all $t>0, b^{\prime}(t) \models_{\mathbb{R}} \Omega$. Also, for all $b^{\prime \prime} \in \bigcup_{\rho} c_{\perp}^{\rho}$, it is $b^{\prime \prime} \models_{\mathbb{R}} \Omega$. 
Now the proof goes by reductio ad absurdum. Let $\omega$ be an bMTL formula of granularity $\rho$ equivalent to $\Omega$. Thus it must be $b_{\top}^{\rho} \models_{\mathbb{R}} \omega, b_{\perp}^{\rho} \forall_{\mathbb{R}} \omega$, and $c_{\perp}^{\rho} \models_{\mathbb{R}} \omega$. So, there exists a $t$ such that $b_{\perp}^{\rho}(t) \not \forall_{\mathbb{R}} \omega$. Let us show that no such $t$ can exist.

Lemma 1 mandates that $b_{\perp}^{\rho}(0) \models_{\mathbb{R}} \omega$, so it must be $t \neq 0$.

If $t>0$, recall that $c_{\perp}^{\rho} \models_{\mathbb{R}} \omega$. This subsumes that $c_{\perp}^{\rho}(u) \models_{\mathbb{R}} \omega$ for all $u>0$, and thus in particular at $t$. However, note that $c_{\perp}^{\rho}(x)=b_{\perp}^{\rho}(x)$ for all $x>0$; since $\omega$ is a future formula, its truth value to the future of 0 cannot change when just one past instant has changed and the future has not changed. So it must be $b_{\perp}^{\rho}(t) \models_{\mathbb{R}} \omega$ : a contradiction.

Let us now assume $t<0$. From Lemma 3 for formula $\omega$, it is either (1) $b_{\top}^{\rho}(t) \models_{\mathbb{R}} \omega$ iff $b_{\perp}^{\rho}(t) \models_{\mathbb{R}} \omega ;$ or $(2) b_{\perp}^{\rho}(t) \models_{\mathbb{R}} \omega$ iff $c_{\perp}^{\rho}(t) \models_{\mathbb{R}} \omega$. However, $b_{\top}^{\rho}(t) \models_{\mathbb{R}} \omega$ and $b_{\perp}^{\rho}(t) \forall_{\mathbb{R}} \omega$, so (1) is false and (2) must be true. Hence, it must be $c_{\perp}^{\rho}(t) \not \forall_{\mathbb{R}} \omega$. But this implies $c_{\perp}^{\rho} \forall_{\mathbb{R}} \omega$, whereas it should be $c_{\perp}^{\rho} \models_{\mathbb{R}} \omega$ since $\omega$ is supposed equivalent to $\Omega$ : a contradiction again.

All in all, no such $\omega$ equivalent to $\Omega$ can exist.

A side remark to better understand the above proofs: the fact that the non-strict formula $\nu=\neg \mathrm{p} \Rightarrow \bigcirc(\neg \mathrm{p})$ distinguishes between the two families of behaviors $\mathcal{B}_{\top}, \mathcal{B}_{\perp}$ does not contradict the proof of Theorem 4 . In fact, what the theorem shows is that $\nu$ cannot be equivalent to $\Omega$ for all behaviors; the fact that it is for some is still possible (in fact notice that $c_{\perp}^{\rho} \forall \forall_{\mathbb{R}} \nu$ ).

\subsection{Matching Less Expressive Than Non-Matching}

This section provides an indirect simple proof that $b \widetilde{\mathrm{MTL}}^{\downarrow} \subset$ b $\widetilde{\mathrm{MTL}}$. To this end we first show the equivalence of non-strict and strict flat matching MTL when restricted to a unary set of propositions.

Lemma 5. Over a unary set of propositions $\mathcal{P}:|\mathcal{P}|=1, \mathrm{MMTL}^{\downarrow}=\mathrm{bMTL}^{\downarrow}$.

Proof. Let us show that any b $\widetilde{\mathrm{MTL}}^{\downarrow}$ formula $\phi=\widetilde{\mathrm{U}}_{I}^{\downarrow}\left(\beta_{1}, \beta_{2}\right)$ has an equivalent bMTL $\downarrow$ formula; without loss of generality assume $0 \notin I$. If $\beta_{1}=\mathrm{p}$ and $\beta_{2}=\neg \mathrm{p}$, or if $\beta_{1}=\neg \mathrm{p}$ and $\beta_{2}=\mathrm{p}$, then $\phi=\perp$ because we are considering matching until; clearly $\perp \in$ bMTL $^{\downarrow}$. If $\beta_{1}=\beta_{2}=\mathrm{p}$ or $\beta_{1}=\beta_{2}=\neg \mathrm{p}$ then $\phi$ holds if and only if $\widetilde{\bigcirc}\left(\beta_{1}\right)$. Now, we already discussed how $\widetilde{\bigcirc}\left(\beta_{1}\right) \equiv \widetilde{\mathrm{U}}_{(0,+\infty)}^{\downarrow}\left(\beta_{1}, \top\right)$. From (2) we can also express it through matching non-strict operators as: $U_{(0,+\infty)}^{\downarrow}\left(\beta_{1}, \top\right) \vee$ $\left(\neg \beta_{1} \wedge \neg \mathrm{U}_{(0,+\infty)}^{\downarrow}\left(\neg \beta_{1}, \top\right)\right)$. So $\phi \in b \mathrm{MTL}^{\downarrow}$ in this case as well. The remaining cases are trivial. Finally, recall that $b \mathrm{MTL}^{\downarrow} \subseteq b \widetilde{\mathrm{MTL}}^{\downarrow}$, hence the lemma.

As a corollary of Lemma 5 we can separate b $\widetilde{\mathrm{MTL}}^{\downarrow}$ and $b \widetilde{\mathrm{MTL}}$ (over general set of propositions).

Theorem 6. $b \widetilde{\mathrm{MTL}}{ }^{\downarrow} \subset b \widehat{\mathrm{MTL}}$.

Proof. Recall that $b \widetilde{\mathrm{MTL}}^{\downarrow} \subseteq b \widetilde{\mathrm{MTL}}$ and bMTL ${ }^{\downarrow} \subseteq b \mathrm{MTL}$. Let us first assume a unary alphabet. Then, it is $b \mathrm{MTL}^{\downarrow}=b \widetilde{\mathrm{MTL}}^{\downarrow} \subseteq b \widetilde{\mathrm{MTL}}$, and also bMTL ${ }^{\downarrow} \subseteq$ $b \mathrm{MTL} \subset \mathrm{b} \widetilde{\mathrm{MTL}}$, the latter from Theorems 2 and 4 . If we assume $b \widetilde{\mathrm{MTL}}^{\downarrow}=$ 
b $\widetilde{\mathrm{MTL}}$ we get: $b \widetilde{\mathrm{MTL}}=\mathrm{b}^{\mathrm{MTL}}{ }^{\downarrow}=($ from Lemma 5$)=b \mathrm{MTL}^{\downarrow} \subseteq$ bMTL $\subset$ b $\widetilde{\mathrm{MTL}}$, a contradiction. So it is $b \widetilde{\mathrm{MTL}}{ }^{\downarrow} \subset b \widetilde{\mathrm{MTL}}$ over unary alphabet, which implies the same holds over generic alphabet.

\subsection{Non-Strict Matching Less Expressive Than Matching}

Section 5 shows that strict flat MTL is strictly more expressive than its nonstrict flat counterpart, when both of them are in their non-matching version. This section shows that the same relation holds if we consider the matching versions of strict and non-strict operators, that is we prove that bMTL ${ }^{\downarrow} \subset$ $\widehat{\text { MTL }}^{\downarrow}$.

First of all, we remark that Lemma 5 entails that any separation proofs for $b \mathrm{MTL}^{\downarrow}$ and $b \widetilde{\mathrm{MTL}}^{\downarrow}$ must consider behaviors over alphabets of size at least two. In fact, we provide such a separation proof by considering behaviors over a binary alphabet, with a technique similar to that of Section 4.1 . The use of a binary alphabet renders the various case analyses even more tedious; thus, for the sake of exposition, we only sketch some details which can be derived from the proof of the previous Section 4.1.

Let us define four families of behaviors $e_{T}^{\rho}, e_{\perp}^{\rho}, f_{T}^{\rho}, f_{\perp}^{\rho}$ over the binary alphabet $\{\mathrm{p}, \mathrm{q}\}$ as follows. First of all: $\mathrm{p} \in e_{\top}^{\rho}(t)$ iff $\mathrm{p} \in b_{\top}^{\rho}(t) ; \mathrm{p} \in e_{\perp}^{\rho}(t)$ iff $\mathrm{p} \in b_{\perp}^{\rho}(t)$; $\mathrm{p} \in f_{\top}^{\rho}(t)$ iff $\mathrm{p} \in c_{\top}^{\rho}(t)$ iff $\mathrm{p} \in f_{\perp}^{\rho}(t)$ iff $\mathrm{p} \in c_{\perp}^{\rho}(t)$. Then, $\mathrm{q} \in e_{\mathrm{T}}^{\rho}(t)$ iff $\mathrm{q} \in e_{\perp}^{\rho}(t)$ iff $\mathrm{q} \in f_{\top}^{\rho}(t)$ iff $\mathrm{q} \in f_{\perp}^{\rho}(t)$ iff $0<t \leq \rho / 4$. The behaviors $e_{\top}^{\rho}, e_{\perp}^{\rho}, f_{\top}^{\rho}, f_{\perp}^{\rho}$ are pictured in Figure 2.

\subsubsection{Initial Satisfiability}

Let us first assume the initial satisfiability semantics; we show that no bMTL $\downarrow$ formula $\phi$ with granularity $\rho$ distinguishes initially between $e_{\top}^{\rho}$ and $e_{\perp}^{\rho}$. To this end, we prove the following.

Lemma 7. For any bMTL ${ }^{\downarrow}$ formula $\phi$ of granularity $\rho$, it is $e_{\top}^{\rho}(0) \models_{\mathbb{R}} \phi$ iff $e_{\perp}^{\rho}(0) \models{ }_{\mathbb{R}} \phi$.

Proof sketch. Note that $\mathrm{q}$ is the same over both $e_{\top}^{\rho}$ and $e_{\perp}^{\rho}$. Therefore, any Boolean combination $\beta$ involving both $\mathrm{p}$ and $\mathrm{q}$ cannot be used to distinguish between $e_{\top}^{\rho}(0)$ and $e_{\perp}^{\rho}(0)$ unless it can do the same using p only. So, a similar case analysis as in the proof of Lemma 1, only lengthier, leads to the present lemma.

As usual, Lemma 7 leads to separation for the initial semantics.

Theorem 8. Under the initial satisfiability semantics, bMTL ${ }^{\downarrow} \subset b \widetilde{\mathrm{MTL}}^{\downarrow}$.

Proof. Let us show that the b $\widetilde{\mathrm{MTL}}^{\downarrow}$ formula $\Sigma^{\downarrow}=\widetilde{\mathrm{U}}_{(0,+\infty)}^{\downarrow}(\mathrm{q}, \mathrm{p})$ has no equivalent $b \mathrm{MTL} \downarrow$ formula. $\Sigma^{\downarrow}$ can distinguish initially between the families of behaviors $\bigcup_{\rho} e_{T}^{\rho}, \bigcup_{\rho} e_{\perp}^{\rho}$, as for any $b \in \bigcup_{\rho} e_{\top}^{\rho}$ and $b^{\prime} \in \bigcup_{\rho} e_{\perp}^{\rho}$, it is $b(0) \models_{\mathbb{R}} \Sigma^{\downarrow}$ and $b^{\prime}(0) \forall \forall_{\mathbb{R}} \Sigma^{\downarrow}$. Let us assume that $\sigma^{\downarrow}$ is a bMTL ${ }^{\downarrow}$ formula of granularity $\rho$ equivalent to $\Sigma^{\downarrow}$. However, from Lemma 7 it follows that $e_{\top}^{\rho}(0) \models_{\mathbb{R}} \sigma^{\downarrow}$ iff $e_{\perp}^{\rho}(0) \models_{\mathbb{R}} \sigma^{\downarrow}$. Therefore, $\sigma^{\downarrow}$ is not equivalent to $\Sigma^{\downarrow}$. 


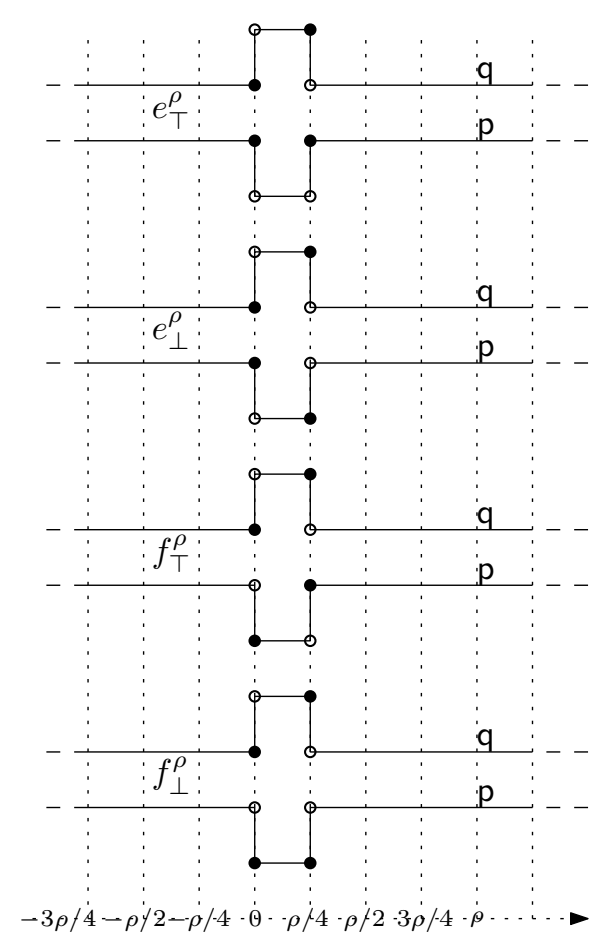

Figure 2: The behaviors $e_{\top}^{\rho}, e_{\perp}^{\rho}, f_{\top}^{\rho}, f_{\perp}^{\rho}$.

\subsubsection{Global Satisfiability}

Also the extension to the global satisfiability semantics is along the usual lines (cmp. Lemma 3).

Lemma 9. For any bMTL ${ }^{\downarrow}$ formula $\phi$ of granularity $\rho$ and any instant $t<0$, it is: $e_{T}^{\rho}(t) \models_{\mathbb{R}} \phi$ iff $e_{\perp}^{\rho}(t) \models_{\mathbb{R}} \phi$, or $e_{\perp}^{\rho}(t) \models_{\mathbb{R}} \phi$ iff $f_{\perp}^{\rho}(t) \models_{\mathbb{R}} \phi$.

Proof sketch. The only way a bMTL ${ }^{\downarrow}$ formula $\phi$ of granularity $\rho$ can distinguish between $e_{\top}^{\rho}(t)$ and $e_{\perp}^{\rho}(t)$ is by "referencing" the instant $\rho / 4$, the only instant at which $e_{\top}^{\rho}$ and $e_{\perp}^{\rho}$ differ. However, if $\phi$ "references" $\rho / 4$ then it cannot reference 0 in any way, because the distance between these two points is less then its granularity. Therefore, its truth value at $t$ cannot change if we change the value of $\mathrm{p}$ at 0 . That is, whenever $e_{T}^{\rho}(t) \nvdash_{\mathbb{R}} \phi$ iff $e_{\perp}^{\rho}(t) \models_{\mathbb{R}} \phi$ it must also be $e_{\perp}^{\rho}(t) \models_{\mathbb{R}} \phi$ iff $f_{\perp}^{\rho}(t) \models_{\mathbb{R}} \phi$.

Finally we extend Theorem 8 to the global satisfiability semantics.

Theorem 10. Under the global satisfiability semantics, bMTL ${ }^{\downarrow} \subset \widehat{b}^{\mathrm{MTL}}{ }^{\downarrow}$.

Proof. Let us show that the $b \widetilde{\mathrm{MTL}}^{\downarrow}$ formula $\Omega^{\downarrow}=\widetilde{\mathrm{U}}_{(0,+\infty)}^{\downarrow}(\mathrm{q}, \mathrm{p}) \vee \bigcirc(\mathrm{q}) \vee \widetilde{O}(\mathrm{p})$ has no equivalent bMTL ${ }^{\downarrow}$ formula. By contradiction, let $\omega^{\downarrow}$ be an equivalent bMTL ${ }^{\downarrow}$ formula, and let $\rho$ be its granularity. Since $e_{\top}^{\rho} \models_{\mathbb{R}} \Omega^{\downarrow}$ and $e_{\perp}^{\rho} \not \models_{\mathbb{R}} \Omega^{\downarrow}$, it must be $e_{\top}^{\rho} \models_{\mathbb{R}} \omega^{\downarrow}$ and $e_{\perp}^{\rho} \nvdash_{\mathbb{R}} \omega^{\downarrow}$. In particular, let $t$ be an instant such that $e_{\perp}^{\rho}(t) \not \nvdash_{\mathbb{R}} \omega^{\downarrow}$ and $e_{\top}^{\rho}(t) \models_{\mathbb{R}}^{\perp} \omega^{\downarrow}$. 
It cannot be $t>\rho / 4$, as $e_{\top}^{\rho}, e_{\perp}^{\rho}$ coincide over $(\rho / 4,+\infty)$ and we are considering future formulas. If $0<t \leq \rho / 4$, let us consider the behavior $f_{\perp}^{\rho}$. Since $f_{\perp}^{\rho} \models_{\mathbb{R}} \Omega^{\downarrow}$ it must be $f_{\perp}^{\rho}(t) \models_{\mathbb{R}} \omega^{\downarrow}$; but it is not possible that the truth value of $\omega^{\downarrow}$ changes by only modifying an instant which is before the current one. If $t=0$ we know that $e_{\top}^{\rho}(t) \models_{\mathbb{R}} \omega^{\downarrow}$ iff $e_{\perp}^{\rho}(t) \models_{\mathbb{R}} \omega^{\downarrow}$ from Lemma 7. So, it must be $t<0$. However in this case it must also be $f_{\perp}^{\rho}(t) \forall_{\mathbb{R}} \omega^{\downarrow}$ from Lemma 9; this is in contradiction with $f_{\perp}^{\rho} \models_{\mathbb{R}} \Omega^{\downarrow}$, which concludes the proof.

\subsection{Non-Strict Matching Less Expressive Than Non-Strict}

Section 4.1 shows that flat non-matching MTL is strictly more expressive than its matching flat counterpart, when both of them are in their strict version. This section shows that the same relation holds if we consider the non-strict versions of matching and non-matching operators, that is we prove that $\mathrm{MTL}^{\downarrow} \subset \mathrm{MTL}$. We sketch a proof similar to the one in the previous Section 4.3, that is based on behaviors over a binary set of propositions 4 All the details can be filled in exactly as in the previous cases.

Let us define four more families of behaviors $g_{\top}^{\rho}, g_{\perp}^{\rho}, h_{\top}^{\rho}, h_{\perp}^{\rho}$ over the alphabet $\{\mathrm{p}, \mathrm{q}\}$ as follows. First of all: $\mathrm{p} \in g_{\top}^{\rho}(t)$ iff $\mathrm{p} \in b_{\top}^{\rho}(t) ; \mathrm{p} \in g_{\perp}^{\rho}(t)$ iff $\mathrm{p} \in b_{\perp}^{\rho}(t)$; $\mathrm{p} \in h_{\top}^{\rho}(t)$ iff $\mathrm{p} \in c_{\top}^{\rho}(t)$ iff $\mathrm{p} \in h_{\perp}^{\rho}(t)$ iff $\mathrm{p} \in c_{\perp}^{\rho}(t)$. Then, $\mathrm{q} \in g_{\top}^{\rho}(t)$ iff $\mathrm{q} \in g_{\perp}^{\rho}(t)$ iff $\mathrm{q} \in h_{\top}^{\rho}(t)$ iff $\mathrm{q} \in h_{\perp}^{\rho}(t)$ iff $t \leq 0$. The behaviors $g_{\top}^{\rho}, g_{\perp}^{\rho}, h_{\top}^{\rho}, h_{\perp}^{\rho}$ are pictured in Figure 3 .

\subsubsection{Initial Satisfiability}

We show that no bMTL ${ }^{\downarrow}$ formula $\phi$ with granularity $\rho$ distinguishes initially between $g_{\top}^{\rho}$ and $g_{\perp}^{\rho}$. To this end we prove the following.

Lemma 11. For any bMTL ${ }^{\downarrow}$ formula $\phi$ of granularity $\rho$, it is $g_{\top}^{\rho}(0) \models_{\mathbb{R}} \phi$ iff $g_{\perp}^{\rho}(0) \models{ }_{\mathbb{R}} \phi$.

Proof sketch. Note that $\mathrm{q}$ is the same over both $e_{\top}^{\rho}$ and $e_{\perp}^{\rho}$. Therefore, any Boolean combination $\beta$ involving both $\mathrm{p}$ and $\mathrm{q}$ cannot be used to distinguish between $g_{\top}^{\rho}(0)$ and $g_{\perp}^{\rho}(0)$ unless it can do the same using $\mathrm{p}$ only. So, a similar case analysis as in the proof of Lemma 1, only longer, leads to the present lemma.

As usual, Lemma 11 leads to separation for the initial semantics.

Theorem 12. Under the initial satisfiability semantics, bMTL ${ }^{\downarrow} \subset b \mathrm{MTL}$.

Proof. Let us show that the bMTL formula $\Sigma^{\prime}=\mathrm{U}_{(0,+\infty)}(\mathrm{q} \vee \neg \mathrm{p}, \mathrm{p})$ has no equivalent $b M T L^{\downarrow}$ formula. $\Sigma^{\prime}$ can distinguish initially between the families of behaviors $\bigcup_{\rho} g_{\top}^{\rho}, \bigcup_{\rho} g_{\perp}^{\rho}$, as for any $b \in \bigcup_{\rho} g_{\top}^{\rho}$ and $b^{\prime} \in \bigcup_{\rho} g_{\perp}^{\rho}$, it is $b(0) \models_{\mathbb{R}} \Sigma^{\prime}$ and $b^{\prime}(0) \forall_{\mathbb{R}} \Sigma^{\prime}$. Let us assume that $\sigma^{\prime}$ is a bMTL ${ }^{\downarrow}$ formula of granularity $\rho$ equivalent to $\Sigma^{\prime}$. However, from Lemma 11 it follows that $g_{\top}^{\rho}(0) \models_{\mathbb{R}} \sigma^{\prime}$ iff $g_{\perp}^{\rho}(0) \models_{\mathbb{R}} \sigma^{\prime}$. Therefore, $\sigma^{\prime}$ is not equivalent to $\Sigma$.

\footnotetext{
${ }^{4}$ We conjecture that a unary alphabet is not enough, but we are currently unable to provide a proof of this fact.
} 


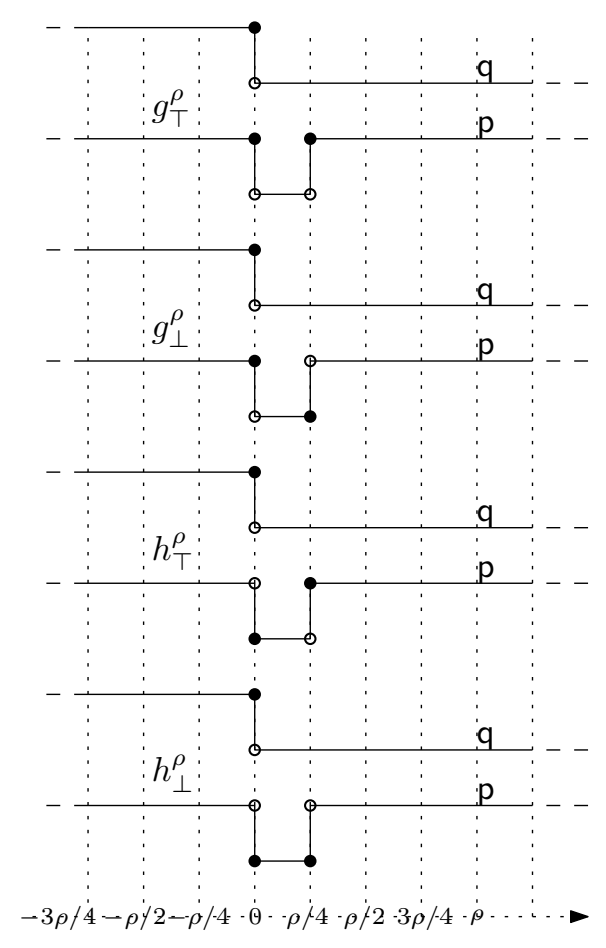

Figure 3: The behaviors $g_{\top}^{\rho}, g_{\perp}^{\rho}, h_{\top}^{\rho}, h_{\perp}^{\rho}$.

\subsubsection{Global Satisfiability}

Also the extension to the global satisfiability semantics is along the usual lines.

Lemma 13. For any bMTL ${ }^{\downarrow}$ formula $\phi$ of granularity $\rho$ and any instant $t<0$, it is: $g_{\top}^{\rho}(t) \models_{\mathbb{R}} \phi$ iff $g_{\perp}^{\rho}(t) \models_{\mathbb{R}} \phi$, or $g_{\perp}^{\rho}(t) \models_{\mathbb{R}} \phi$ iff $h_{\perp}^{\rho}(t) \models_{\mathbb{R}} \phi$.

Proof sketch. The only way a bMTL $\mathrm{M}^{\downarrow}$ formula $\phi$ of granularity $\rho$ can distinguish between $g_{\top}^{\rho}(t)$ and $g_{\perp}^{\rho}(t)$ is by "referencing" the instant $\rho / 4$, the only instant at which $g_{\top}^{\rho}$ and $g_{\perp}^{\rho}$ differ. However, if $\phi$ "references" $\rho / 4$ then it cannot reference 0 in any way, because the distance between these two points is less then its granularity. Therefore, its truth value at $t$ cannot change if we change the value of $\mathrm{p}$ at 0 . That is, whenever $g_{\top}^{\rho}(t) \nvdash_{\mathbb{R}} \phi$ iff $g_{\perp}^{\rho}(t) \models_{\mathbb{R}} \phi$ it must also be $g_{\perp}^{\rho}(t) \models_{\mathbb{R}} \phi$ iff $h_{\perp}^{\rho}(t) \models_{\mathbb{R}} \phi$.

Finally we extend Theorem 12 to the global satisfiability semantics.

Theorem 14. Under the global satisfiability semantics, bMTL ${ }^{\downarrow} \subset$ bMTL.

Proof. Let us show that the formula $\Omega^{\prime}=U_{(0,+\infty)}(q \vee \neg p, p) \vee \bigcirc(\neg p) \vee \widetilde{\bigcirc}(p)$ has no equivalent bMTL formula. By contradiction, let $\omega^{\prime}$ be an equivalent bMTL ${ }^{\downarrow}$ formula, and let $\rho$ be its granularity. Since $g_{\top}^{\rho} \models_{\mathbb{R}} \Omega^{\prime}$ and $g_{\perp}^{\rho} \nvdash_{\mathbb{R}} \Omega^{\prime}$, it must be $g_{\top}^{\rho} \models_{\mathbb{R}} \omega^{\prime}$ and $g_{\perp}^{\rho} \nvdash_{\mathbb{R}} \omega^{\prime}$. In particular, let $t$ be an instant such that $g_{\perp}^{\rho}(t) \not \nvdash_{\mathbb{R}} \omega^{\prime}$ and $g_{\top}^{\rho}(t) \models_{\mathbb{R}} \omega^{\prime}$.

It cannot be $t>\rho / 4$, as $g_{\top}^{\rho}, g_{\perp}^{\rho}$ coincide over $(\rho / 4,+\infty)$ and we are considering future formulas. If $0<t \leq \rho / 4$, let us consider the behavior $h_{\perp}^{\rho}$. Since 


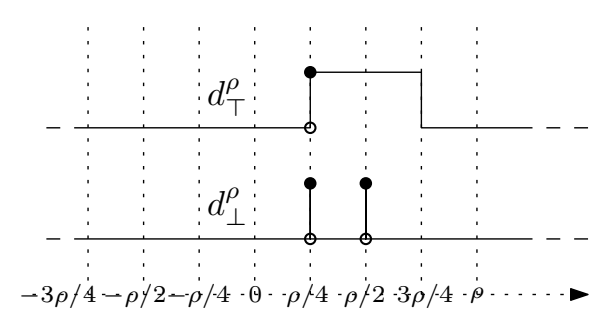

Figure 4: The behaviors $d_{\top}^{\rho}, d_{\perp}^{\rho}$.

$h_{\perp}^{\rho} \models_{\mathbb{R}} \Omega^{\prime}$ it must be $h_{\perp}^{\rho}(t) \models_{\mathbb{R}} \omega^{\prime}$; but it is not possible that the truth value of $\omega^{\prime}$ changes by only modifying an instant which is before the current one. If $t=0$ we know that $g_{\top}^{\rho}(t) \models_{\mathbb{R}} \omega^{\prime}$ iff $g_{\perp}^{\rho}(t) \models_{\mathbb{R}} \omega^{\prime}$ from Lemma 11. So, it must be $t<0$. However in this case it must be $h_{\perp}^{\rho}(t) \forall_{\mathbb{R}} \omega^{\prime}$ from Lemma 13; this is in contradiction with $h_{\perp}^{\rho} \models_{\mathbb{R}} \Omega^{\prime}$, which concludes the proof.

\subsection{Flat Less Expressive Than Nesting}

This section shows that $b \widetilde{\mathrm{MTL}} \subset$ MTL through a technique similar to that used in Section 4.1 .

Let us define the following families of behaviors. For any given $\rho>0$, let $d_{\top}^{\rho}$ and $d_{\perp}^{\rho}$ be defined as follows: $\mathrm{p} \in d_{\top}^{\rho}(t)$ iff $\rho / 4 \leq t<3 \rho / 4$; and $\mathrm{p} \in d_{\perp}^{\rho}(t)$ iff $t=\rho / 4$ or $t=\rho / 2$. The behaviors $d_{\top}^{\rho}, d_{\perp}^{\rho}$ are pictured in Figure 4. Also, for any $u \in \mathbb{R}, \epsilon>0$, let $d_{u}^{\epsilon}$ be defined as follows: $\mathrm{p} \in d_{u}^{\epsilon}(t)$ iff $u \leq t<u+\epsilon$ or $t=\rho / 4$ or $t=\rho / 2$.

\subsubsection{Initial Satisfiability}

Let us first assume the initial satisfiability semantics and show that no b $\widetilde{\mathrm{MTL}}$ formula of granularity $\rho$ can distinguish initially between $d_{\top}^{\rho}, d_{\perp}^{\rho}$. To this end we prove the following.

Lemma 15. For any b $\widetilde{\mathrm{MTL}}$ formula $\phi$ of granularity $\rho$, it is $d_{\top}^{\rho}(0) \models_{\mathbb{R}} \phi$ iff $d_{\perp}^{\rho}(0) \models{ }_{\mathbb{R}} \phi$.

Proof. As usual the proof is by induction on the structure of $\phi$.

- $\phi=\beta$, where $\beta$ is a Boolean combination of $\mathrm{p}$ and $\neg \mathrm{p}$. Since $d_{\top}^{\rho}(0)=$ $d_{\perp}^{\rho}(0)$ this case follows trivially. Also note that $\beta$ is equivalent to one of $\mathrm{p}, \neg \mathrm{p}, \top, \perp$ over both $d_{\top}^{\rho}$ and $d_{\perp}^{\rho}$.

- $\phi=\neg \phi^{\prime}$. By inductive hypothesis it is $d_{\top}^{\rho}(0) \models_{\mathbb{R}} \phi^{\prime}$ iff $d_{\perp}^{\rho}(0) \models_{\mathbb{R}} \phi^{\prime}$, so clearly $d_{\top}^{\rho}(0) \models_{\mathbb{R}} \neg \phi^{\prime}$ iff $d_{\perp}^{\rho}(0) \models_{\mathbb{R}} \neg \phi^{\prime}$.

- $\phi=\phi_{1} \wedge \phi_{2}$. By inductive hypothesis, $d_{\top}^{\rho}(0) \models_{\mathbb{R}} \phi_{1}$ iff $d_{\perp}^{\rho}(0) \models_{\mathbb{R}} \phi_{1}$, and $d_{\top}^{\rho}(0) \models_{\mathbb{R}} \phi_{2}$ iff $d_{\perp}^{\rho}(0) \models_{\mathbb{R}} \phi_{2}$. Thus $d_{\top}^{\rho}(0) \models_{\mathbb{R}} \phi_{1} \wedge \phi_{2}$ iff $\overline{d_{\perp}^{\rho}}(0) \models_{\mathbb{R}} \phi_{1} \wedge \phi_{2}$.

- $\phi=\widetilde{\mathrm{U}}_{I}\left(\beta_{1}, \beta_{2}\right)$, with $I=\langle l, u\rangle$ and: (1) $l=k_{1} \rho$ for some $k_{1} \in \mathbb{N}$; and $(2)$ $u=k_{2} \rho$ or $u=+\infty$, for some $k_{1} \leq k_{2} \in \mathbb{N}$. Note that we can assume 
$0 \notin I$ without loss of generality, as $\widetilde{\mathbf{U}}_{[0, u\rangle}\left(\beta_{1}, \beta_{2}\right) \equiv \beta_{2} \vee \widetilde{\mathbf{U}}_{(0, u\rangle}\left(\beta_{1}, \beta_{2}\right)$. We also assume that $I$ is non-empty; this is also without loss of generality.

We consider several cases for $\beta_{1}, \beta_{2}$.

- If $\beta_{1} \equiv \beta_{2} \equiv \top$ then clearly $d_{\top}^{\rho}(0) \models_{\mathbb{R}} \phi$ and $d_{\perp}^{\rho}(0) \models_{\mathbb{R}} \phi$.

- If $\beta_{2} \equiv \perp$ then clearly $d_{\top}^{\rho}(0) \not \nvdash_{\mathbb{R}} \phi$ and $d_{\perp}^{\rho}(0) \not \nvdash_{\mathbb{R}} \phi$.

- If $\beta_{1} \equiv \perp$ then $d_{\top}^{\rho}(0) \not \nvdash_{\mathbb{R}} \phi$ and $d_{\perp}^{\rho}(0) \not \nvdash_{\mathbb{R}} \phi$, as $0 \notin I$.

- If $\beta_{1} \equiv \top$ and $\beta_{2}$ is one of $\mathrm{p}$ or $\neg \mathrm{p}$, we have $\phi \equiv \diamond_{I}\left(\beta_{2}\right)$.

If $l=0$, then $u>l$, which entails $u \geq \rho$. Any interval of the form $\langle 0, \rho\rangle$ encompasses both instants where $p$ holds and instants where $\neg p$ holds. Thus, $d_{\top}^{\rho}(0) \models_{\mathbb{R}} \phi$ and $d_{\perp}^{\rho}(0) \models_{\mathbb{R}} \phi$ in this case. If $l>0$ then $l \geq \rho$. Since $d_{\top}^{\rho}$ and $d_{\perp}^{\rho}$ are the same for all $t \geq \rho$, it is $d_{\top}^{\rho}(0) \models_{\mathbb{R}} \phi$ iff $d_{\perp}^{\rho}(0) \models_{\mathbb{R}} \phi$.

- If $\beta_{1} \equiv \mathrm{p}$, then $\phi$ does not hold unless $\widetilde{O}(\mathrm{p})$ holds (still because $0 \notin I)$. Since it is $\widetilde{\bigcirc}(\neg p)$ for both behaviors at 0 , it is $d_{\top}^{\rho}(0) \not \nvdash_{\mathbb{R}} \phi$ and $d_{\perp}^{\rho}(0) \forall_{\mathrm{R}} \phi$.

- If $\beta_{1} \equiv \neg \mathrm{p}$ and $\beta_{2} \equiv \top$ or $\beta_{2} \equiv \neg \mathrm{p}$, then $\phi$ is equivalent to $\widetilde{\bigcirc}(\neg \mathrm{p})$ (still because $0 \notin I)$. So $d_{\top}^{\rho}(0) \models_{\mathbb{R}} \phi$ and $d_{\perp}^{\rho}(0) \models_{\mathbb{R}} \phi$.

- If $\beta_{1} \equiv \neg \mathrm{p}$ and $\beta_{2} \equiv \mathrm{p}$, and $I=(0, u\rangle$, then it is clear that $\widetilde{U}_{I}(\neg \mathrm{p}, \mathrm{p})$ is true at 0 , since $\mathrm{p}$ switches to true at $\rho / 4$ right-continuously in $d_{T}^{\rho}$ and point-wisely in $d_{\perp}^{\rho}$. So $d_{\top}^{\rho}(0) \models_{\mathbb{R}} \phi$ and $d_{\perp}^{\rho}(0) \models_{\mathbb{R}} \phi$ in this case. In instead $l \neq 0$ then $l \geq \rho$. Thus $\phi$ does not hold at 0 unless $\square_{(0, \rho)}(\neg p)$ holds at the same instant. But $p$ takes both truth values within the interval $(0, \rho)$, thus $d_{\top}^{\rho}(0) \nvdash_{\mathbb{R}} \phi$ and $d_{\perp}^{\rho}(0) \nvdash_{\mathbb{R}} \phi$.

Lemma 15 leads straightforwardly to the desired separation result.

Theorem 16. Under the initial satisfiability semantics, b $\widetilde{\mathrm{MTL}} \subset \mathrm{MTL}$.

Proof. Let us show that the MTL formula $\Xi=\diamond_{(0,+\infty)}(\bigcirc(\mathrm{p}))$ has no equivalent bMTL formula. $\Xi$ can distinguish initially between the families of behaviors $\bigcup_{\rho \in \mathbb{R}_{>0}} d_{\top}^{\rho}, \bigcup_{\rho \in \mathbb{R}_{>0}} d_{\perp}^{\rho}$, as for any $b \in \bigcup_{\rho \in \mathbb{R}_{>0}} d_{\top}^{\rho}$ and $b^{\prime} \in \bigcup_{\rho \in \mathbb{R}_{>0}} d_{\perp}^{\rho}$, it is

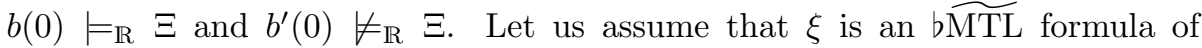
granularity $\rho$ equivalent to $\Xi$. However, from Lemma 1 it follows that $d_{\top}^{\rho}(0) \models_{\mathbb{R}}$ $\xi$ iff $d_{\perp}^{\rho}(0) \models_{\mathbb{R}} \xi$. Therefore, $\xi$ is not equivalent to $\Xi$.

\subsubsection{Global Satisfiability}

Let us now extend Theorem 16 to the global satisfiability semantics. To this end, we first prove the following non-distinguishability result.

Lemma 17. For any $b \widetilde{\mathrm{MTL}}$ formula $\phi$ of granularity $\rho$ and any instant $t \leq \rho / 2$, there exist $u, \epsilon$ such that $[u, u+\epsilon) \subseteq[\rho / 4,3 \rho / 4)$ and $d_{\perp}^{\rho}(t) \models_{\mathbb{R}} \phi$ iff $d_{u}^{\epsilon}(t) \models_{\mathbb{R}} \phi$.

Proof. The proof is by induction on the structure of $\phi$; throughout $t \leq \rho / 2$. Recall that for $u=\rho / 4$ and $\epsilon=\rho / 2$ we have $d_{\top}^{\rho}=d_{u}^{\epsilon}$. 
- $\phi=\beta$. If $t \leq \rho / 4$ or $t=\rho / 2$, note that $d_{\top}^{\rho}(t)=d_{\perp}^{\rho}(t)$, so this case follows trivially. Otherwise $\rho / 4<t<\rho / 2$ and take $u=\rho / 2$ and $\epsilon=\rho / 4$; in this case $d_{\perp}^{\rho}(t)=d_{u}^{\epsilon}$.

- $\phi=\neg \phi^{\prime}$. By inductive hypothesis, there exist $u, \epsilon$ such that $d^{\prime}=d_{u}^{\epsilon}$ and $d^{\prime}(t) \models_{\mathbb{R}} \phi^{\prime}$ iff $d_{\perp}^{\rho}(t) \models_{\mathbb{R}} \phi^{\prime}$. Thus also $d^{\prime}(t) \models_{\mathbb{R}} \phi$ iff $d_{\perp}^{\rho}(t) \models_{\mathbb{R}} \phi$.

- $\phi=\widetilde{U}_{I}\left(\beta_{1}, \beta_{2}\right)$, with $I=\langle a, b\rangle, a=k_{1} \rho, b=k_{2} \rho \geq a$ or $b=+\infty$, for $k_{1}, k_{2}$ nonnegative integers. Also, without loss of generality, we assume $0 \notin I$. We consider several cases for $\beta_{1}, \beta_{2}$.

- If $\beta_{1} \equiv \beta_{2} \equiv \top$, then $d_{\top}^{\rho}(t) \models_{\mathbb{R}} \phi$ and $d_{\perp}^{\rho}(t) \models_{\mathbb{R}} \phi$.

- If $\beta_{1} \equiv \perp$ or $\beta_{2} \equiv \perp$, then $d_{\top}^{\rho}(t) \forall_{\mathbb{R}} \phi$ and $d_{\perp}^{\rho}(t) \forall_{\mathbb{R}} \phi$.

- If $\beta_{1} \equiv \top$ and $\beta_{2}$ is one of $\mathrm{p}, \neg \mathrm{p}$, we have $\phi \equiv \diamond_{I}\left(\beta_{2}\right)$. We further consider several cases.

* If $a=b=k \rho$ and $t+k \rho \neq \rho / 4$ and $t+k \rho \neq \rho / 2$, let us discuss the value of $t+k \rho$. If $\rho / 2<t+k \rho \leq 3 \rho / 4$ then let $u=\rho / 4$ and $\epsilon=t+k \rho-\rho / 4$; if $t+k \rho<\rho / 4$ or $t+k \rho>3 \rho / 4$ let $u=\rho / 4$ and $\epsilon=\rho / 2$; if $\rho / 4<t+k \rho<\rho / 2$ let $u=\rho / 2$ and $\epsilon=\rho / 4$. Then note that, in all cases, $d_{\perp}^{\rho}(t+k \rho)=d_{u}^{\epsilon}(t+k \rho)$, therefore $d_{\perp}^{\rho}(t) \models_{\mathbb{R}} \phi$ iff $d_{u}^{\epsilon}(t) \models_{\mathbb{R}} \phi$.

* If $a=b=k \rho$ and $t+k \rho=\rho / 4$, let $u=\rho / 2$ and $\epsilon=\rho / 4$. Then note that $d_{\perp}^{\rho}(t+k \rho)=d_{u}^{\epsilon}(t+k \rho)$, therefore $d_{\perp}^{\rho}(t) \models_{\mathbb{R}} \phi$ iff $d_{u}^{\epsilon}(t) \models{ }_{\mathbb{R}} \phi$.

* If $a=b=k \rho$ and $t+k \rho=\rho / 2$, let $u=\rho / 4$ and $\epsilon=\rho / 4$. Then note that $d_{\perp}^{\rho}(t+k \rho)=d_{u}^{\epsilon}(t+k \rho)$, therefore $d_{\perp}^{\rho}(t) \models_{\mathbb{R}} \phi$ iff $d_{u}^{\epsilon}(t) \models{ }_{\mathbb{R}} \phi$.

$*$ If $a<b$ then $b-a \geq \rho$. If $t+a<\rho / 2$ or $t+a \geq 3 \rho / 4$ or $t+a=\rho / 2 \wedge a \in I$, notice that the interval $I$ of size $\geq \rho$ is such that $d_{\top}^{\rho}(t) \models_{\mathbb{R}} \vartheta_{\langle a, b\rangle}\left(\beta_{2}\right)$ iff $d_{\perp}^{\rho}(t) \models_{\mathbb{R}} \vartheta_{\langle a, b\rangle}\left(\beta_{2}\right)$. Therefore, $d_{\top}^{\rho}(t) \models_{\mathbb{R}} \phi$ iff $d_{\perp}^{\rho}(t) \models_{\mathbb{R}} \phi$.

If instead $t+a=\rho / 2 \wedge a \notin I$, let $u=\rho / 4$ and $\epsilon=\rho / 4$, and note that $d_{\perp}^{\rho}(t) \models_{\mathbb{R}} \phi$ iff $d_{u}^{\epsilon}(t) \models_{\mathbb{R}} \phi$ in this case since $\mathrm{p}$ is constantly false within $I$.

Finally, assume $\rho / 4<t+a<\rho / 2$ and let $u=\rho / 2$ and $\epsilon=\rho / 4$. Note that $d_{\perp}^{\rho}(t) \models_{\mathbb{R}} \phi$ and $d_{u}^{\epsilon}(t) \models_{\mathbb{R}} \phi$, as both $\mathrm{p}$ and $\neg \mathrm{p}$ hold within $I$.

- If $\beta_{1} \equiv \beta_{2}$, then $\phi$ holds iff $\widetilde{\bigcirc}\left(\beta_{2}\right)$. So, if $t<\rho / 4$ or $\rho / 4<t<\rho / 2$, clearly $d_{\perp}^{\rho}(t) \models_{\mathbb{R}} \phi$ iff $d_{\top}^{\rho}(t) \models_{\mathbb{R}} \phi$. If $t=\rho / 4$ instead, we pick $u=\rho / 2$ and $\epsilon=\rho / 4$, and if $t=\rho / 2$, we pick $u=\rho / 4$ and $\epsilon=\rho / 4$. In both cases $d_{\perp}^{\rho}(t) \models_{\mathbb{R}} \phi$ iff $d_{u}^{\epsilon}(t) \models_{\mathbb{R}} \phi$.

- If $\beta_{1} \equiv \mathrm{p}, \beta_{2} \equiv \neg \mathrm{p}$, and $t<\rho / 4$, then surely $d_{\perp}^{\rho}(t) \forall_{\mathbb{R}} \phi$ and $d_{\mathrm{T}}^{\rho}(t) \not \forall_{\mathbb{R}} \phi$. If $\rho / 4 \leq t<\rho / 2$ instead, we pick $u=\rho / 2$ and $\epsilon=\rho / 4$, so that again $d_{\perp}^{\rho}(t) \forall_{\mathbb{R}} \phi$ and $d_{u}^{\epsilon}(t) \forall_{\mathbb{R}} \phi$. Finally, if $t=\rho / 2$ we pick $u=\rho / 4$ and $\epsilon=\rho / 4$, so that $d_{\perp}^{\rho}(t) \forall_{\mathbb{R}} \phi$ and $d_{u}^{\epsilon}(t) \forall_{\mathbb{R}} \phi$ in this case as well.

- Similarly, if $\beta_{1} \equiv \neg \mathrm{p}, \beta_{2} \equiv \mathrm{p}$, and $t<\rho / 4$ then surely $d_{\perp}^{\rho}(t) \models_{\mathbb{R}} \phi$ and $d_{\top}^{\rho}(t) \models_{\mathbb{R}} \phi$. If $\rho / 4 \leq t<\rho / 2$ instead, we pick $u=\rho / 2$ and $\epsilon=\rho / 4$, 
so that again $d_{\perp}^{\rho}(t) \models_{\mathbb{R}} \phi$ and $d_{u}^{\epsilon}(t) \models_{\mathbb{R}} \phi$. Finally, if $t=\rho / 2$ we pick $u=\rho / 4$ and $\epsilon=\rho / 4$, so that $d_{\perp}^{\rho}(t) \nvdash_{\mathbb{R}} \phi$ and $d_{u}^{\epsilon}(t) \nvdash_{\mathbb{R}} \phi$ in this case.

Overall, notice that it is $b_{\perp}^{\rho}(t) \models_{\mathbb{R}} \phi$ iff $d_{u}^{\epsilon}(t) \models_{\mathbb{R}} \phi$ and $u=\rho / 4$ and $\epsilon>\rho / 4$ unless: (a) $u=\rho / 2, \epsilon \leq \rho / 4$ and $\rho / 4 \leq t+k \rho<\rho / 2$ for some nonnegative $k$; or (b) $u=\rho / 4, \epsilon \leq \rho / 4$ and $t+h \rho=\rho / 2$ for some nonnegative integer $h$.

- $\phi=\phi_{1} \wedge \phi_{2}$. In the proof of this case, note how we can "lift" restrictions on the value of $t$ to the conjunction itself, to go with the inductive hypothesis.

If $d_{\perp}^{\rho}(t) \models_{\mathbb{R}} \phi_{i}$ iff $d_{u_{i}}^{\epsilon_{i}}(t) \models_{\mathbb{R}} \phi_{i}$ with $u_{i}=\rho / 4$ and $\epsilon_{i}>\rho / 4$ for both $i=1,2$, then it is not difficult to check that $d_{\perp}^{\rho}(t) \models_{\mathbb{R}} \phi$ iff $d_{u^{\prime}}^{\epsilon^{\prime}}(t) \models_{\mathbb{R}} \phi$ with $u^{\prime}=\rho / 4$ and $\epsilon^{\prime}=\min \left(\epsilon_{1}, \epsilon_{2}\right)>\rho / 4$ and we are done.

Otherwise, consider the case $u_{i}=\rho / 4$ and $\epsilon_{i}>\rho / 4$ for some $i$, and $u_{j}=\rho / 2, \epsilon_{j} \leq \rho / 4, \rho / 4 \leq t+k \rho<\rho / 2$ for $j \neq i$. Then we can state that $d_{\perp}^{\rho}(t) \models_{\mathbb{R}} \phi$ iff $d_{u^{\prime}}^{\epsilon^{\prime}}(t) \models_{\mathbb{R}} \phi$ with $u^{\prime}=\rho / 2$ and $\epsilon^{\prime}=\min \left(\epsilon_{j}, \epsilon_{i}-\rho / 4\right)$, and $\rho / 4 \leq t+k \rho<\rho / 2$. We are done, since clearly $\epsilon^{\prime} \leq \rho / 4$.

If $u_{i}=\rho / 4$ and $\epsilon_{i}>\rho / 4$ for some $i$, and $u_{j}=\rho / 4, \epsilon_{j} \leq \rho / 4, t+h \rho=\rho / 2$ for $j \neq i$. Then we can state that $d_{\perp}^{\rho}(t) \models_{\mathbb{R}} \phi$ iff $d_{u^{\prime}}^{\epsilon^{\prime}}(t) \models_{\mathbb{R}} \phi$ with $u^{\prime}=\rho / 4$ and $\epsilon^{\prime}=\epsilon_{j} \leq \rho / 4$, and $t+h \rho=\rho / 2$.

Finally, consider the case $u_{i}=\rho / 2, \epsilon_{i} \leq \rho / 4, \rho / 4 \leq t+k \rho<\rho / 2$ for some $i$, and $u_{j}=\rho / 4, \epsilon_{j} \leq \rho / 4, t+h \rho=\rho / 2$ for $j \neq i$. Substituting $t=\rho / 2-h \rho$ in $\rho / 4 \leq t+k \rho<\rho / 2$ and simplifying, we get $-\rho / 4 \leq(k-h) \rho<0$. Since $k-h$ is an integer, $(k-h) \rho<0$ implies $k-h \leq-1$ and thus $(k-h) \rho \leq-\rho$, which is incompatible with $(k-h) \rho \geq-\rho / 4$. Hence this case is closed by contradiction.

Through Lemma 17 we can extend Theorem 16 to the global satisfiability semantics.

Theorem 18. Under the global satisfiability semantics, b $\widetilde{\mathrm{MTL}} \subset \mathrm{MTL}$.

Proof. Let us show that the MTL formula:

$$
\Gamma=\diamond_{[0,+\infty)}(\bigcirc(\mathrm{p})) \vee \square_{[0,+\infty)}(\neg \mathrm{p}) \vee \mathrm{U}_{(0,+\infty)}\left(\neg \mathrm{p},\left(\mathrm{p} \wedge \square_{(0,+\infty)}(\neg \mathrm{p})\right)\right)
$$

has no equivalent b $\widetilde{\mathrm{MTL}}$ formula.

To the contrary, assume that there exists a b $\widetilde{\mathrm{MTL}}$ formula $\gamma$ equivalent to $\Omega$. Let $\rho$ be the granularity of $\gamma$, and let us consider the behaviors $d_{\top}^{\rho}$ and $d_{\perp}^{\rho}$. Since you can check that $d_{\top}^{\rho} \models_{\mathbb{R}} \Gamma$ and $d_{\perp}^{\rho} \nvdash_{\mathbb{R}} \Gamma$ (in fact, a non-strict until is used), it must be $d_{\top}^{\rho} \models_{\mathbb{R}} \gamma$ and $d_{\perp}^{\rho} \nvdash_{\mathbb{R}} \gamma$, so $\gamma$ must be false somewhere on $d_{\perp}^{\rho}$. Let $t$ be any instant such that $d_{\perp}^{\rho}(t) \nvdash_{\mathbb{R}} \gamma$.

If $t>\rho / 2$, then let us consider the behavior $d^{\prime}=d_{\rho / 4}^{t-\rho / 4}$; clearly $d^{\prime} \models_{\mathbb{R}} \Gamma$, so it must be $d^{\prime} \models_{\mathbb{R}} \gamma$ and, in particular, $d^{\prime}(t) \models_{\mathbb{R}} \gamma$. However, $d^{\prime}$ and $d_{\perp}^{\rho}$ coincide over the interval $[t,+\infty)$, therefore $d_{\perp}^{\rho}(t)=d^{\prime}(t) \forall_{\mathbb{R}} \gamma$. This shows that it must be $t \leq \rho / 2$.

If $t \leq \rho / 2$, Lemma 17 requires that there exists a $d^{\prime \prime}=d_{u}^{\epsilon}$ such that: $[u, u+$ $\epsilon) \subseteq[\rho / 4,3 \rho / 4)$ and $d_{\perp}^{\rho}(t) \models_{\mathbb{R}} \gamma$ iff $d^{\prime \prime}(t) \models_{\mathbb{R}} \gamma$. Thus $d^{\prime \prime}(t) \nvdash_{\mathbb{R}} \gamma$ in our 
case, which entails $d^{\prime \prime} \nvdash_{\mathbb{R}} \gamma$ as well. On the contrary, $d^{\prime \prime} \models_{\mathbb{R}} \Gamma$, since the sub-formula $\diamond_{[0,+\infty)}(\bigcirc(\mathrm{p}))$ holds throughout $(-\infty, u+\epsilon)$ and the other subformula $\square_{[0,+\infty)}(\neg \mathrm{p}) \vee \mathrm{U}_{(0,+\infty)}\left(\neg \mathrm{p},\left(\mathrm{p} \wedge \square_{(0,+\infty)}(\neg \mathrm{p})\right)\right)$ holds afterward, over $[u+\epsilon,+\infty)$. This contradicts the supposed equivalence between $\gamma$ and $\Gamma$.

\section{$5 \quad$ Nesting MTL over Zeno Behaviors}

This section re-considers some of the expressiveness results for nesting formulas of Section 3 when Zeno behaviors are allowed as interpretation structures.

A simplified MTL subset. First of all it is convenient to introduce some additional notational simplifications. Henceforth, we denote the intervals $(0, \delta)$, $(0, \delta],[\delta, \delta]$, and $(\delta,+\infty)$ as $<\delta, \leq \delta,=\delta$, and $>\delta$, respectively.

Then, $\phi \stackrel{\delta}{\equiv} \phi^{\prime}$ denotes that the truth value of $\phi$ coincides with that of $\phi^{\prime}$ over behavior $b_{\delta}$ (or $b_{\delta}^{\mathcal{Z}}$ when we write $\phi \stackrel{\mathcal{Z}}{=} \phi^{\prime}$ ). That is, $\phi \stackrel{\delta}{=} \phi^{\prime}$ iff $\forall t \in \mathbb{T}:\left(b_{\delta}(t) \models_{\mathbb{T}}\right.$ $\phi$ iff $\left.b_{\delta}(t) \models_{\mathbb{T}} \phi^{\prime}\right)$, and $\phi \stackrel{\underline{\underline{Z}}}{=} \phi^{\prime}$ iff $\forall t \in \mathbb{T}:\left(b_{\delta}^{\mathcal{Z}}(t) \models_{\mathbb{T}} \phi\right.$ iff $\left.b_{\delta}^{\mathcal{Z}}(t) \models_{\mathbb{T}} \phi^{\prime}\right)$.

Let us show that the following MTL fragment is expressively complete for MTL formulas with granularity $\delta$.

$$
\phi::=\mathrm{p}\left|\mathrm{U}_{<\delta}\left(\phi_{1}, \phi_{2}\right)\right| \mathrm{U}_{=\delta}\left(\phi_{1}, \phi_{2}\right)\left|\mathrm{U}_{>\delta}\left(\phi_{1}, \phi_{2}\right)\right| \neg \phi \mid \phi_{1} \wedge \phi_{2}
$$

To this end, it suffices to consider an MTL formula $\phi=\mathrm{U}_{I}\left(\phi_{1}, \phi_{2}\right)$ of granularity $\delta$; therefore $I=\langle a \delta, b \delta\rangle$ for some $a, b \in \mathbb{N}$. Let us consider several cases.

- $I=(0, \delta): \phi \equiv \mathrm{U}_{<\delta}\left(\phi_{1}, \phi_{2}\right)$.

- $I=(0, \delta]: \phi \equiv \mathrm{U}_{<\delta}\left(\phi_{1}, \phi_{2}\right) \vee \mathrm{U}_{=\delta}\left(\phi_{1}, \phi_{2}\right)$.

- $I=(0, b \delta), b>1: \phi \equiv \bigvee_{k=0}^{b-2} \diamond_{=k \delta}\left(\mathrm{U}_{\leq \delta}\left(\phi_{1}, \phi_{2}\right)\right) \vee \diamond_{=(b-1) \delta}\left(\mathrm{U}_{<\delta}\left(\phi_{1}, \phi_{2}\right)\right)$.

- $I=(0, b \delta], b>1: \phi \equiv \bigvee_{k=0}^{b-1} \diamond_{=k \delta}\left(\mathrm{U}_{\leq \delta}\left(\phi_{1}, \phi_{2}\right)\right)$.

- $I=[0, b \delta\rangle, b \geq 0: \phi \equiv \mathrm{U}_{(0, b \delta\rangle}\left(\phi_{1}, \phi_{2}\right) \vee\left(\phi_{1} \wedge \phi_{2}\right)$.

- $I=(a \delta, b \delta), b>a>0: \phi \equiv \square_{[0, a \delta]}\left(\phi_{1}\right) \wedge \diamond_{=a \delta}\left(\mathrm{U}_{(0,(b-a) \delta)}\left(\phi_{1}, \phi_{2}\right)\right)$.

- We omit the remaining cases as they are all similar to the previous ones.

\subsection{Non-Strict Less Expressive Than Strict}

This section proves that the equivalence between MTL and $\widetilde{M T L}$ of [FR07a] does not hold if we allow Zeno behaviors. Intuitively, the equivalence (2) does not hold if Zeno behaviors are disallowed, because it may be that $\neg \widetilde{\bigcirc}(p) \wedge \neg \widetilde{\bigcirc}(\neg p)$ for some item $\mathrm{p}$ over a Zeno behavior, thus failing to satisfy (1). In fact, we can prove that MTL $\subset \widetilde{\text { MTL }}$ for Zeno behaviors.

To this end, we define behaviors $b_{\delta}, b_{\delta}^{\mathcal{Z}}$ over $\mathcal{P}=\{\mathrm{p}\}$, for all $\delta>0 . b_{\delta}$ is defined as: $\mathrm{p} \in b_{\delta}(t)$ iff $t=k \delta / 2$ for some $k \in \mathbb{Z}$. $b_{\delta}^{\mathcal{Z}}$ is defined as: $\mathrm{p} \in b_{\delta}^{\mathcal{Z}}(t)$ iff $t=\left(k+2^{-n}\right) \delta$, for some $k \in \mathbb{Z}, n \in \mathbb{N}$. Clearly, for all $t \in \mathbb{T}, \mathrm{p} \in b_{\delta}(t)$ implies $\mathrm{p} \in b_{\delta}^{\mathcal{Z}}(t)$; moreover, notice that $b_{\delta}^{\mathcal{Z}}$ has Zeno behavior to the right of any instant $k \delta$. 


\subsubsection{Primitive Behavior of MTL Formulas}

This section shows that the behavior of any MTL formula over $b_{\delta}$ and $b_{\delta}^{\mathcal{Z}}$ is very simple, as it coincides with one of $p, \neg p, \top, \perp$. This fact will be used in the following to tackle the separation proof.

Lemma 19. The truth value of any MTL formulas $\phi$ of granularity $\delta$ over $b_{\delta}$ coincides with one of $\mathrm{p}, \neg \mathrm{p}, \top, \perp$. That is, one of the following holds.

$$
\begin{gathered}
\phi \stackrel{\delta}{\equiv} \mathrm{p} \\
\phi \stackrel{\delta}{=} \neg \mathrm{p} \\
\phi \stackrel{\delta}{=} \top \\
\phi \stackrel{\delta}{=} \perp
\end{gathered}
$$

Proof. The proof goes by induction on the structure of the formula $\phi$.

Base case. The base case is for formulas $\phi$ that do not use operator at all, i.e., $\phi$ is one of $p, \top$, or $\perp$. The proof is trivial.

Inductive case. In the inductive case, we assume the statement of the lemma to hold for all $\phi$ nesting up to $k-1$ operators (both temporal operators and propositional connectives), and we prove it for nesting depth equal to $k$ as well. We consider five cases for $\phi$.

- $\phi=\neg \psi$. By inductive hypothesis, the truth value of $\psi$ over $b_{\delta}$ coincides with one of $p, \neg p, T$, or $\perp$. Therefore, the truth value of $\neg \psi$ over $b_{\delta}$ coincides with $\neg \mathrm{p}, \mathrm{p}, \perp$, or $\top$, respectively.

- $\phi=\psi_{1} \wedge \psi_{2}$. By inductive hypothesis, the truth values of $\psi_{1}, \psi_{2}$ over $b_{\delta}$ coincide with one of $\mathrm{p}, \neg \mathrm{p}, \top$, or $\perp$. Therefore, it is easy to check that the the truth value of $\psi_{1} \wedge \psi_{2}$ over $b_{\delta}$ coincides with one of $\mathrm{p}, \neg \mathrm{p}, \top$, or $\perp$.

- $\phi=\mathrm{U}_{<\delta}\left(\psi_{1}, \psi_{2}\right)$. By inductive hypothesis, we consider several cases.

- If the truth value of $\psi_{1}$ coincides with $\perp$ or $\mathrm{p}$, then so does the truth value of $\phi$. In fact, $b_{\delta}(t) \models_{\mathbb{T}} \phi$ requires in particular that there exists a $u \in(t, t+\delta)$ such that for all $v \in[t, u)$ it is $b(v) \models_{\mathbb{T}} \psi_{1}$. But $\perp$ never holds, and $\mathrm{p}$ holds only at isolated points.

- If the truth value of $\psi_{1}$ coincides with $\top, \phi$ is equivalent to $\nabla_{<\delta}\left(\psi_{2}\right)$. Moreover, by inductive hypothesis $\psi_{2}$ coincides with one of $\mathrm{p}, \neg \mathrm{p}, \top$, or $\perp$. Since $\mathrm{p}$ holds periodically every $\delta / 2$ instants over $b_{\delta}$, and $\neg \mathrm{p}$ holds everywhere except at isolated points, it is simple to realize that the truth value of $\vartheta_{<\delta}\left(\psi_{2}\right)$ coincides either with $\top$ or with $\perp$.

- If the truth value of $\psi_{1}$ coincides with $\neg \mathrm{p}, \phi$ is equivalent to $\mathrm{U}_{<\delta}\left(\neg \mathrm{p}, \psi_{2}\right)$. If $\psi_{2} \equiv \perp$, then obviously $\phi \equiv \perp$. If $\psi_{2} \equiv \top$, then $\phi \equiv \bigcirc(\neg \mathrm{p})$; it is simple to check that $\neg \mathrm{p} \Leftrightarrow \bigcirc(\neg \mathrm{p})$ everywhere, as $\neg p$ holds over open intervals. Therefore, $\phi \equiv \neg p$ in this case. If $\psi_{2} \equiv \mathrm{p}$ or $\psi_{2} \equiv \neg \mathrm{p}$, then similarly one can show that $\phi \equiv \neg \mathrm{p}$.

- $\phi=\mathrm{U}_{=\delta}\left(\psi_{1}, \psi_{2}\right)$. By inductive hypothesis, we consider several cases. We omit the proofs whenever they are all similar to the previous $\mathrm{U}_{<\delta}$ case. 
- If the truth value of $\psi_{1}$ coincides with $\perp$ or $\mathrm{p}$, then so does the truth value of $\phi$.

- If the truth value of $\psi_{1}$ coincides with $\top, \phi$ is equivalent to $\diamond_{=\delta}\left(\psi_{2}\right)$. Moreover, by inductive hypothesis $\psi_{2}$ coincides with one of $\mathrm{p}, \neg \mathrm{p}$, $T$, or $\perp$. Since $b_{\delta}(t) \models_{\mathbb{T}} \phi$ iff $b_{\delta}(t+\delta) \models_{\mathbb{T}} \psi_{2}$, and $b_{\delta}(t) \models_{\mathbb{T}} \mathrm{p}$ iff $b_{\delta}(t+\delta) \models_{\mathbb{T}} \mathrm{p}$, it is simple to realize that the truth value of $\phi$ also coincides with one of $p, \neg p, \top$, or $\perp$.

- If the truth value of $\psi_{1}$ coincides with $\neg \mathrm{p}, \phi$ is equivalent to $\square_{[0, \delta)}(\neg \mathrm{p}) \wedge$ $\vartheta_{=\delta}\left(\psi_{2}\right)$. However, $\square_{[0, \delta)}(\neg p)$ is always false, as any interval of length $\delta$ also includes instants where $\mathrm{p}$ is true. Therefore, $\phi \equiv \perp$ in this case.

- $\phi=\mathrm{U}_{>\delta}\left(\psi_{1}, \psi_{2}\right)$. By inductive hypothesis, we consider several cases. We omit the proofs whenever they are all similar to the previous cases $U_{<\delta}$ and $\mathrm{U}_{=\delta}$.

- If the truth value of $\psi_{1}$ coincides with $\perp$ or $\mathrm{p}$, then so does the truth value of $\phi$.

- If the truth value of $\psi_{1}$ coincides with $\top, \phi$ is equivalent to $\nabla_{>\delta}\left(\psi_{2}\right)$. Moreover, by inductive hypothesis $\psi_{2}$ coincides with one of $\mathrm{p}, \neg \mathrm{p}, \top$, or $\perp$. Since $\mathrm{p}$ holds periodically every $\delta / 2$ instants over $b_{\delta}$, and $\neg p$ holds everywhere except at isolated points, it is simple to realize that the truth value of $\nabla_{>\delta}\left(\psi_{2}\right)$ coincides either with $\top$ or with $\perp$.

- If the truth value of $\psi_{1}$ coincides with $\neg p, \phi$ implies in particular that $\square_{[0, \delta+\epsilon)}(\neg p)$ for some $\epsilon>0$. However, $\square_{[0, \delta+\epsilon)}(\neg p)$ is false for any $\epsilon>0$, as any interval of length greater than $\delta$ also includes instants where $\mathrm{p}$ is true. Therefore, $\phi \equiv \perp$ in this case.

Lemma 20. The truth value of any MTL formulas $\phi$ of granularity $\delta$ over $b_{\delta}^{\mathcal{Z}}$ coincides with one of $\mathrm{p}, \neg \mathrm{p}, \top, \perp$. That is, one of the following holds.

$$
\begin{gathered}
\phi \stackrel{\underline{\underline{\underline{Z}}}}{\mathrm{p}} \\
\phi \underline{\underline{\underline{\underline{Z}}}} \neg \mathrm{p} \\
\phi \underline{\underline{\underline{\underline{z}}}} \top \\
\phi \stackrel{\underline{\underline{\underline{Z}}}}{ } \perp
\end{gathered}
$$

Proof. The proof goes by induction on the structure of the formula $\phi$. We consider only the relevant cases, that is those that differ from the proof of Lemma 19. In particular, we only focus on a part of the inductive cases.

- $\phi=\mathrm{U}_{<\delta}\left(\psi_{1}, \psi_{2}\right)$. By inductive hypothesis, we consider several cases.

- If the truth value of $\psi_{1}$ coincides with $\perp$ or $\mathrm{p}$, then so does the truth value of $\phi$. In fact, $b_{\delta}^{\mathcal{Z}}(t) \models_{\mathrm{T}} \phi$ requires in particular that there exists a $u \in(t, t+\delta)$ such that for all $v \in[t, u)$ it is $b(v) \models_{\mathbb{T}} \psi_{1}$. But $\perp$ never holds, and $\mathrm{p}$ holds only at isolated points, unless it accumulates (at any point $k \delta$, for some integer $k$ ). In particular, notice that any interval of the form $[k \delta, k \delta+\epsilon)$ for some $k \in \mathbb{Z}, \epsilon \in \mathbb{T}_{>0}$ includes both instants where $\mathrm{p}$ is true and instants where it is false. 
- If the truth value of $\psi_{1}$ coincides with $T, \phi$ is equivalent to $\nabla_{<\delta}\left(\psi_{2}\right)$. Moreover, by inductive hypothesis $\psi_{2}$ coincides with one of $\mathrm{p}, \neg \mathrm{p}$, $\top$, or $\perp$. Since $p$ holds at least every $\delta / 2$ instants over $b_{\delta}^{\mathcal{Z}}$, and $\neg p$ holds "infinitely often", it is simple to realize that the truth value of $\vartheta_{<\delta}\left(\psi_{2}\right)$ coincides either with $\top$ or with $\perp$.

- If the truth value of $\psi_{1}$ coincides with $\neg p, \phi$ is equivalent to $\mathrm{U}_{<\delta}\left(\neg \mathrm{p}, \psi_{2}\right)$. If $\psi_{2} \equiv \perp$, then obviously $\phi \equiv \perp$. If $\psi_{2} \equiv \top$, then $\phi \equiv \bigcirc(\neg \mathrm{p})$. Notice that $b_{\delta}^{\mathcal{Z}}(t) \models_{\mathbb{T}} \neg \mathrm{p} \Leftrightarrow \bigcirc(\neg \mathrm{p})$ for all $t \neq k \delta$ for some integer $k$, and $b_{\delta}^{\mathcal{Z}}(k \delta) \models_{\mathbb{T}} \mathrm{p} \wedge \neg \bigcirc(\neg \mathrm{p})$. Therefore, $\phi \equiv \neg \mathrm{p}$ in this case. If $\psi_{2} \equiv \mathrm{p}$ or $\psi_{2} \equiv \neg \mathrm{p}$, then similarly one can show that $\phi \equiv \neg p$.

- $\phi=\mathrm{U}_{=\delta}\left(\psi_{1}, \psi_{2}\right)$. By inductive hypothesis, we consider several cases. We omit the proofs whenever they are all similar to the previous $\mathrm{U}_{<\delta}$ case.

- If the truth value of $\psi_{1}$ coincides with $\perp$ or $\mathrm{p}$, then so does the truth value of $\phi$.

- If the truth value of $\psi_{1}$ coincides with $T$, $\phi$ is equivalent to $\nabla_{=\delta}\left(\psi_{2}\right)$. Moreover, by inductive hypothesis $\psi_{2}$ coincides with one of $\mathrm{p}, \neg \mathrm{p}$, $\top$, or $\perp$. Since $b_{\delta}^{\mathcal{Z}}(t) \models_{\mathbb{T}} \phi$ iff $b_{\delta}^{\mathcal{Z}}(t+\delta) \models_{\mathbb{T}} \psi_{2}$, and $b_{\delta}^{\mathcal{Z}}(t) \models_{\mathrm{T}} \mathrm{p}$ iff $b_{\delta}^{\mathcal{Z}}(t+\delta) \models_{\mathbb{T}} \mathrm{p}$, it is simple to realize that the truth value of $\phi$ also coincides with one of $\mathrm{p}, \neg \mathrm{p}, \top$, or $\perp$.

- If the truth value of $\psi_{1}$ coincides with $\neg p, \phi$ is equivalent to $\square_{[0, \delta)}(\neg p) \wedge$ $\vartheta_{=\delta}\left(\psi_{2}\right)$. However, $\square_{[0, \delta)}(\neg p)$ is always false, as any interval of length $\delta$ also includes instants where $\mathrm{p}$ is true. Therefore, $\phi \equiv \perp$ in this case.

- $\phi=\mathrm{U}_{>\delta}\left(\psi_{1}, \psi_{2}\right)$. By inductive hypothesis, we consider several cases. We omit the proofs whenever they are all similar to the previous cases $U_{<\delta}$ and $\mathrm{U}_{=\delta}$.

- If the truth value of $\psi_{1}$ coincides with $\perp$ or $\mathrm{p}$, then so does the truth value of $\phi$.

- If the truth value of $\psi_{1}$ coincides with $\top, \phi$ is equivalent to $\nabla_{>\delta}\left(\psi_{2}\right)$. Moreover, by inductive hypothesis $\psi_{2}$ coincides with one of $\mathrm{p}, \neg \mathrm{p}, \mathrm{T}$, or $\perp$. Since $\mathrm{p}$ holds periodically at least every $\delta / 2$ instants over $b_{\delta}^{\mathcal{Z}}$, and $\neg p$ holds "infinitely often", it is simple to realize that the truth value of $\nabla_{>\delta}\left(\psi_{2}\right)$ coincides either with $T$ or with $\perp$.

- If the truth value of $\psi_{1}$ coincides with $\neg \mathrm{p}, \phi$ implies in particular that $\square_{[0, \delta+\epsilon)}(\neg p)$ for some $\epsilon>0$. However, $\square_{[0, \delta+\epsilon)}(\neg p)$ is false for any $\epsilon>0$, as any interval of length greater than $\delta$ also includes instants where $\mathrm{p}$ is true. Therefore, $\phi \equiv \perp$ in this case.

By comparing the proofs of Lemmas 19 and 20, any MTL formula has a truth value over $b_{\delta}$ that coincides with the same primitive proposition (i.e., $\mathrm{p}, \neg \mathrm{p}, \top, \perp)$ as it coincides over $b_{\delta}^{\mathcal{Z}}$.

Lemma 21. For any MTL formula $\phi$ of granularity $\delta$, the truth value of $\phi$ over $b_{\delta}$ coincides with the same atomic proposition as it coincides over $b_{\delta}^{\mathcal{Z}}$. In other 
words, with reference to the previously defined formulas:

$$
\begin{array}{lll}
(12) & \text { iff } & (16) \\
(13) & \text { iff } & (17) \\
(14) & \text { iff } & (18) \\
(15) & \text { iff } & (19)
\end{array}
$$

Proof. The proof is again by induction on the structure of $\phi$. In the proof, we write $x$ to denote any of $p, \neg p, T, \perp$. As the proof is clearly symmetric with respect to $b_{\delta}$ and $b_{\delta}^{\mathcal{Z}}$, we just show the $\Rightarrow$ implication.

The base case $(\phi=\mathrm{p}, \top, \perp)$ is trivial, so let us just consider the inductive case.

- $\phi=\neg \psi$. Obviously, $\phi \stackrel{\delta}{=} \times$ implies that $\psi \stackrel{\delta}{=} \neg x$; by inductive hypothesis, also $\psi \stackrel{\mathcal{Z}}{=} \neg x$; therefore $\phi \stackrel{\mathcal{Z}}{=}$ x.

- $\phi=\psi_{1} \wedge \psi_{2}$. Let $\psi_{1} \stackrel{\delta}{=} \mathrm{x}_{1}$ and $\psi_{2} \stackrel{\delta}{=} \mathrm{x}_{2}$; by inductive hypothesis $\psi_{1} \stackrel{\underline{\underline{Z}}}{=} \mathrm{x}_{1}$ and $\psi_{2} \stackrel{\stackrel{\mathcal{Z}}{=}}{\mathrm{x}_{2}}$. Therefore $\phi \stackrel{\mathcal{Z}}{=} \mathrm{x}_{1} \wedge \mathrm{x}_{2}$.

- $\phi=\mathrm{U}_{<\delta}\left(\psi_{1}, \psi_{2}\right)_{\dot{z}}$ Let $\psi_{1} \stackrel{\delta}{=} \mathrm{x}_{1}$ and $\psi_{2} \stackrel{\delta}{=} \mathrm{x}_{2}$; by inductive hypothesis $\psi_{1} \stackrel{\underline{\underline{Z}}}{=} \mathrm{x}_{1}$ and $\psi_{2} \stackrel{\underline{\underline{\mathcal{Z}}}}{=} \mathrm{x}_{2}$. Next, consider the case $\mathrm{x}_{1}=\top$ and $\mathrm{x}_{2} \neq \perp$. Then, it is easy to check that $\phi \equiv T$ on both $b_{\delta}$ and $b_{\delta}^{\mathcal{Z}}$. Otherwise, let $\mathrm{x}_{2}=\perp$ or $\mathrm{x}_{1} \neq \top$. Then, obviously $\phi \equiv \perp$ on both $b_{\delta}$ and $b_{\delta}^{\mathcal{Z}}$.

- $\phi=\mathrm{U}_{=\delta}\left(\psi_{1}, \psi_{2}\right)$. Let $\psi_{1} \stackrel{\delta}{=} \mathrm{x}_{1}$ and $\psi_{2} \stackrel{\delta}{=} \mathrm{x}_{2}$; by inductive hypothesis $\psi_{1} \stackrel{\underline{\underline{Z}}}{=} \mathrm{x}_{1}$ and $\psi_{2} \stackrel{\underline{\underline{Z}}}{\underline{\underline{n}}} \mathrm{x}_{2}$. Next, consider the case $\mathrm{x}_{1} \neq \top$ or $\mathrm{x}_{2}=\perp$. Then, it is easy to check that $\phi \equiv \perp$ on both $b_{\delta}$ and $b_{\delta}^{\mathcal{Z}}$. Otherwise, let $\mathrm{x}_{1}=T=\mathrm{x}_{2}$; then clearly $\phi \stackrel{\delta}{=} T \stackrel{\underline{\underline{\underline{Z}}}}{=} \phi$. Another case is $\mathrm{x}_{1}=\top$ and $\mathrm{x}_{2}=\mathrm{p}$; then $\phi$ reduces to $\nabla_{=d}(\mathrm{p})$, a translation of length $\delta$. Now, recall that both $b_{\delta}$ and $b_{\delta}^{\mathcal{Z}}$ are periodic of period $\delta$; as a consequence, $\vartheta_{=d}(\mathbf{p})$ is equivalent to just $\mathrm{p}$ on both $b_{\delta}$ and $b_{\delta}^{\mathcal{Z}}$. Finally, we have the case $\mathrm{x}_{1}=\top$ and $\mathrm{x}_{2}=\neg \mathrm{p}$; similarly, thanks to the periodicity of $b_{\delta}$ and $b_{\delta}^{\mathcal{Z}}, \phi$ is equivalent to $\neg p$ on both behaviors.

- $\phi=\mathrm{U}_{>\delta}\left(\psi_{1}, \psi_{2}\right)_{\dot{z}}$ Let $\psi_{1} \stackrel{\delta}{=} \mathrm{x}_{1}$ and $\psi_{2} \stackrel{\delta}{=} \mathrm{x}_{2}$; by inductive hypothesis $\psi_{1} \stackrel{\underline{\underline{\mathcal{Z}}}}{\underline{\underline{x}}} \mathrm{x}_{1}$ and $\psi_{2} \stackrel{\underline{\underline{\mathcal{Z}}}}{=} \mathrm{x}_{2}$. Next, consider the case $\mathrm{x}_{1}=\top$ and $\mathrm{x}_{2} \neq \perp$. Then, it is easy to check that $\phi \equiv T$ on both $b_{\delta}$ and $b_{\delta}^{Z}$. Otherwise, let $\mathrm{x}_{2}=\perp$ or $\mathrm{x}_{1} \neq \top$. Then, obviously $\phi \equiv \perp$ on both $b_{\delta}$ and $b_{\delta}^{\mathcal{Z}}$.

An immediate consequence of the previous lemma is that, at any instant where the values $b_{\delta}(t)$ and $b_{\delta}^{\mathcal{Z}}(t)$ coincide, the truth values of any formula $\phi$ also coincide.

Corollary 22. For any MTL formula $\phi$ of granularity $\delta$, and all $k \in \mathbb{Z}$ :

$$
b_{\delta}(k \delta) \models_{\mathbb{T}} \phi \quad \text { iff } \quad b_{\delta}^{\mathcal{Z}}(k \delta) \models_{\mathbb{T}} \phi
$$

Proof. First of all notice that, for all $k \in \mathbb{Z}, b_{\delta}(k \delta)=b_{\delta}^{\mathcal{Z}}(k \delta)$. Then, the corollary follows from Lemma 21. 


\subsubsection{Non-Strict Is Less Expressive Than Strict}

Building upon the lemmas of the previous section, we can prove the desired separation result.

Theorem 23. If Zeno behaviors are allowed, MTL $\subset \widetilde{\mathrm{MTL}}$.

Proof. Let us consider the two families of behaviors: $\mathcal{N}=\left\{b_{\delta} \mid \delta \in \mathbb{Q}_{>0}\right\}$ and $\mathcal{Z}=\left\{b_{\delta}^{\mathcal{Z}} \mid \delta \in \mathbb{Q}_{>0}\right\}$.

Initial satisfiability. First, let $\models_{\mathbb{T}}$ denote "initial satisfiability". The

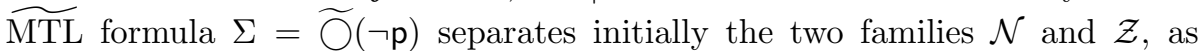
$b(0) \models_{\mathbb{T}} \Sigma$ for all $b \in \mathcal{N}$ and $b^{\prime}(0) \nvdash_{\mathbb{T}} \Sigma$ for all $b^{\prime} \in \mathcal{Z}$.

On the contrary, let $\phi$ be any MTL formula with rational endpoints, and let $\delta$ be its granularity. Then, $\mathcal{N} \ni b_{\delta}(0) \models_{\mathrm{T}} \phi$ iff $\mathcal{Z} \ni b_{\delta}^{\mathcal{Z}}(0) \models_{\mathrm{T}} \phi$ by Corollary 22, so no MTL formula separates initially the two families. This implies that the MTL formula $\Sigma$ has no initially equivalent formula in MTL.

Global satisfiability. Now, let $\models_{\mathrm{T}}$ denote "global satisfiability". The $\widetilde{\text { MTL }}$ formula $\Sigma^{\prime}=\mathrm{p} \Rightarrow \widetilde{\bigcirc}(\neg p)$ separates globally the two families $\mathcal{N}$ and $\mathcal{Z}$, as $b(t) \models_{\mathbb{T}} \Sigma^{\prime}$ for all $t \in \mathbb{T}$ and for all $b \in \mathcal{N}$, and $b^{\prime}(t) \nvdash_{\mathbb{T}} \Sigma^{\prime}$ for some $t=k \delta$, and for all $b^{\prime} \in \mathcal{Z}$.

On the contrary, let $\phi$ be any MTL formula with rational endpoints, and let $\delta$ be its granularity. For the sake of contradiction, assume that $b(t) \models_{\mathbb{T}} \phi$ for all $t \in \mathbb{T}$ and for all $b \in \mathcal{N}$, and that $b^{\prime}(t) \nvdash_{\mathbb{T}} \phi$ for some $t$, and for all $b^{\prime} \in \mathcal{Z}$. Now, in particular, $b_{\delta} \models_{\mathbb{T}} \phi ;$ a fortiori, $b_{\delta}(0) \models_{\mathbb{T}} \phi^{\prime}$ where $\phi^{\prime}=\square_{[0,+\infty)}(\phi)$. Similarly, it must be $b_{\delta}^{\mathcal{Z}} \nvdash_{\mathbb{T}} \phi$. A little reasoning should convince us that this implies $b_{\delta}^{\mathcal{Z}}(0) \nvdash_{\mathbb{T}} \phi^{\prime}$. In fact, $b_{\delta}^{\mathcal{Z}} \nvdash_{\mathbb{T}} \phi$ means that there exists a $t \in \mathbb{T}$ such that $b_{\delta}^{\mathcal{Z}}(t) \nvdash_{\mathbb{T}} \phi . t$ may be greater than, equal to, or less than 0. However, $b_{\delta}^{\mathcal{Z}}$ is periodic with period $\delta$; this implies that $b_{\delta}^{\mathcal{Z}}(t) \models_{\mathbb{T}} \alpha$ iff $b_{\delta}^{\mathcal{Z}}(t+k \delta) \models_{\mathrm{T}} \alpha$, for all formulas $\alpha, t \in \mathbb{T}, k \in \mathbb{Z}$. Therefore, if there exists a $t \in \mathbb{T}$ such that $b_{\delta}^{\mathcal{Z}}(t) \forall_{\mathbb{T}} \phi$, then also there exists a $t^{\prime} \geq 0$ such that $b_{\delta}^{\mathcal{Z}}\left(t^{\prime}\right) \nvdash_{\mathbb{T}} \phi$. The last formula implies that $b_{\delta}^{\mathcal{Z}}(0) \forall_{\mathbb{T}} \phi^{\prime}$.

Now, notice that the formula $\phi^{\prime}$ is of the same granularity as $\phi$, that is $\delta$. Moreover, $b_{\delta}(0) \models_{\mathbb{T}} \phi^{\prime}$ and $b_{\delta}^{\mathcal{Z}}(0) \nvdash_{\mathbb{T}} \phi^{\prime}$. This contradicts Corollary 22; therefore $\phi$ does not globally separate the two families of behaviors. Since $\phi$ is generic, the $\widehat{\mathrm{MTL}}$ formula $\Sigma^{\prime}$ has no globally equivalent formula in MTL.

\subsection{Non-Strict Matching Less Expressive Than Strict Match- ing}

If we reconsider all the theorems of Section 5.1, and the corresponding proofs, we notice that they still stand if we consider the matching variants of the nonstrict and strict until. In other words, the same proofs provide a separation between MTL ${ }^{\downarrow}$ and $\widetilde{\mathrm{MTL}^{\downarrow}}{ }^{\downarrow}$.

Theorem 24. If Zeno behaviors are allowed, $\mathrm{MTL}^{\downarrow} \subset \widetilde{\mathrm{MTL}} \widetilde{\downarrow}^{\downarrow}$. 


\subsection{Matching as Expressive as Non-Matching}

Finally, we turn to the comparison of the matching and non-matching variants when Zeno behaviors are allowed. We can show that the equivalences holding for non-Zeno behaviors are still valid with Zeno behaviors, only with slightly longer proofs 5 Also, this holds regardless of whether we are considering the strict or non-strict variants of until. Therefore, we are able to show that $\widetilde{\mathrm{MTL}}^{\downarrow}=\widetilde{\mathrm{MTL}}$ and MTL $^{\downarrow}=$ MTL over generic behaviors.

In order to present the new proofs, it is convenient to introduce the following notation: $b(t) \models_{\mathbb{R}}$ acc $(\phi)$ denotes the fact that the truth value of formula $\phi$ accumulates to the right of instant $t$; in other words, $\phi$ has a Zeno behavior to the right of $t$ over $b$. Formally, this means that for all $\epsilon>0$ there exists $0<\nu_{1}, \nu_{2}<\epsilon$ such that $b\left(t+\nu_{1}\right) \models_{\mathbb{R}} \phi$ and $b\left(t+\nu_{2}\right) \models_{\mathbb{R}} \neg \phi$; that is $\phi$ takes both truth values infinitely often to the right of $t$. Recall that, if $b(t) \models_{\mathbb{R}}$ acc $(\phi)$ then $b(t) \models \mathbb{R} \neg \widetilde{\bigcirc}(\phi) \wedge \neg \widetilde{\bigcirc}(\neg \phi)$.

\subsubsection{Strict Variant}

Let us start with the proof of equivalence of the matching and non-matching variants of strict until. To this end, let us prove that the equivalences (8-11) still hold when Zeno behaviors are allowed. In fact, we just have to consider explicitly the case of Zeno behaviors in the proofs.

Let us first consider Formula 8 , which we repeat here for clarity 6

$$
\begin{aligned}
\widetilde{\mathrm{U}}_{(0, b\rangle}\left(\phi_{1}, \phi_{2}\right) \equiv & \widetilde{\mathrm{U}}_{(0, b\rangle}^{\downarrow}\left(\phi_{1}, \phi_{2}\right) \\
& \vee\left(\diamond_{(0, b\rangle}\left(\phi_{2}\right) \wedge \widetilde{\bigcirc}\left(\phi_{1}\right) \wedge \widetilde{\mathrm{R}}_{(0, b\rangle}^{\downarrow}\left(\phi_{2}, \bigcirc\left(\phi_{1}\right)\right)\right.
\end{aligned}
$$

Proof of Formula 8 with generic behaviors. Let us start with the $\Rightarrow$ direction, and let $t$ be the current instant. Assume that there exists a $d \in(0, b)$ such that $b(t+d) \models_{\mathbb{R}} \phi_{2}$ and for all $u \in(t, t+d)$ it is $b(u) \models_{\mathbb{R}} \phi_{1}$. If also $b(t+d) \models_{\mathbb{R}} \phi_{1}$ or $b(t) \models_{\mathbb{R}} \widetilde{\bigcirc}\left(\phi_{2}\right)$ then $b(t) \models_{\mathbb{R}} \widetilde{U}_{(0, b\rangle}^{\downarrow}\left(\phi_{1}, \phi_{2}\right)$, so assume that $b(t+d) \models_{\mathbb{R}} \neg \phi_{1}$ and $b(t) \models_{\mathbb{R}} \neg \widetilde{\bigcirc}\left(\phi_{2}\right)$. If also $b(t) \models_{\mathbb{R}}$ acc $\left(\phi_{2}\right)$, then there exists a $0<\nu<d$ such that $b(t+\nu) \models_{\mathbb{R}} \phi_{2}$; therefore $\phi_{2} \wedge \phi_{1}$ holds at $t+\nu \in(t, t+d) \subseteq(t, t+b\rangle$ which entails $b(t) \models_{\mathbb{R}} \widetilde{U}_{(0, b\rangle}^{\downarrow}\left(\phi_{1}, \phi_{2}\right)$.

Otherwise, $\phi_{2}$ does not accumulate to the right of $t$, and thus $b(t) \models_{\mathbb{R}}$ $\widetilde{\bigcirc}\left(\neg \phi_{2}\right)$ in this case. Therefore, it is well-defined $d^{\prime}$, the smallest instant in $(t, t+b\rangle$ such that $b\left(d^{\prime}\right) \models_{\mathbb{R}} \phi_{2} \vee \widetilde{\bigcirc}\left(\phi_{2}\right) \vee$ acc $\left(\phi_{2}\right)$; note that $d^{\prime} \leq t+d$. Note that clearly $b(t) \models_{\mathbb{R}} \widetilde{\bigcirc}\left(\phi_{1}\right)$ and $b(t) \models_{\mathbb{R}} \diamond_{(0, b\rangle}\left(\phi_{2}\right)$.

Let us first consider the case $b\left(d^{\prime}\right) \models_{\mathbb{R}} \phi_{2}$, and let $v \in(t, t+b\rangle$. If $v \geq t+d$, there exists a $w=t+d$ such that $w \in(t, v]$ and $b(w) \models_{\mathbb{R}} \phi_{2}$. If $v<t+d$ instead, then $v \in(t, t+d)$ so $b(v) \models_{\mathbb{R}} \bigcirc\left(\phi_{1}\right)$. All this shows that $b(t) \models_{\mathbb{R}}$ $\widetilde{\mathrm{R}}_{(0, b\rangle}^{\downarrow}\left(\phi_{2}, \bigcirc\left(\phi_{1}\right)\right)$, which completes the proof in this case.

Let us now consider the case $b\left(d^{\prime}\right) \models_{\mathbb{R}} \neg \phi_{2} \wedge \widetilde{\bigcirc}\left(\phi_{2}\right)$; hence $d^{\prime}<t+d$. So, let us assume that $\phi_{2}$ holds over the interval $\left(d^{\prime}, d^{\prime}+\epsilon\right)$ for some $\epsilon>0$. Now, let

\footnotetext{
${ }^{5}$ We are grateful to anonymous referee \# 4, who reviewed the conference version of this paper [FR07b], whose detailed comments prompted us to realize this fact.

${ }^{6}$ Compare the following proof with that for non-Zeno behaviors, in Section 3.2
} 
$v \in(t, t+b\rangle$. If $v>d^{\prime}$, there exists a $w=d^{\prime}+\min \left(v-d^{\prime}, \epsilon\right) / 2$ such that $w \in(t, v]$ and $b(w) \models_{\mathbb{R}} \phi_{2}$. If $v \leq d^{\prime}$ instead, then $v \in\left(t, d^{\prime}\right] \subset(t, t+d)$ so $b(v) \models_{\mathbb{R}} \bigcirc\left(\phi_{1}\right)$, as $\phi_{1}$ holds throughout $(t, t+d)$. All this shows that $b(t) \models_{\mathbb{R}} \widetilde{R}_{(0, b\rangle}^{\downarrow}\left(\phi_{2}, \bigcirc\left(\phi_{1}\right)\right)$, which completes the proof in this case as well.

Finally, assume $b\left(d^{\prime}\right) \models_{\mathbb{R}} \neg \phi_{2} \wedge \neg \widetilde{\bigcirc}\left(\phi_{2}\right) \wedge$ acc $\left(\phi_{2}\right)$; in this case $d^{\prime}<t+d$ as well. Since $\phi_{2}$ accumulates to the right of $d^{\prime}$, there exists a $0<\nu<(t+d)-d^{\prime}$ such that $b(t+d+\nu) \models_{\mathbb{R}} \phi_{2}$; therefore $\phi_{2} \wedge \phi_{1}$ holds at $t+d+\nu \in(t, t+d) \subseteq$ $(t, t+b\rangle$ which entails $b(t) \models_{\mathbb{R}} \widetilde{U}_{(0, b\rangle}^{\downarrow}\left(\phi_{1}, \phi_{2}\right)$.

Let us now consider the $\Leftarrow$ direction, and let $t$ be the current instant. If $b(t) \models_{\mathbb{R}} \widetilde{\mathrm{U}}_{(0, b\rangle}^{\downarrow}\left(\phi_{1}, \phi_{2}\right)$ clearly also $b(t) \models_{\mathbb{R}} \widetilde{\mathrm{U}}_{(0, b\rangle}\left(\phi_{1}, \phi_{2}\right)$ a fortiori. So let us assume that $\widetilde{\mathrm{U}}_{(0, b)}^{\downarrow}\left(\phi_{1}, \phi_{2}\right)$ is false at $t$; note that this subsumes that $b(t) \models_{\mathbb{R}}$ $\neg \widetilde{\bigcirc}\left(\phi_{2} \wedge \phi_{1}\right)$ and that $b(t) \models_{\mathbb{R}} \widetilde{\bigcirc}\left(\phi_{1}\right)$, i.e., $\phi_{1}$ holds over an interval $(t, t+\epsilon)$ for some $\epsilon>0$.

If $b(t) \models_{\mathbb{R}}$ acc $\left(\phi_{2}\right)$, then there exists a $0<\nu<\min (\epsilon, b)$ such that $b(t+\nu) \models_{\mathbb{R}}$ $\phi_{2}$; so $b(t) \models_{\mathbb{R}} \widetilde{\mathrm{U}}_{(0, b\rangle}\left(\phi_{1}, \phi_{2}\right)$ a fortiori. Otherwise, if $\phi_{2}$ does not accumulate, $\widetilde{\mathrm{O}}\left(\neg \phi_{2}\right)$ must hold at $t$. Therefore, it is well-defined $u$, the smallest instant in $(t, t+b\rangle$ such that $b(u) \models_{\mathbb{R}} \phi_{2} \vee \widetilde{O}\left(\phi_{2}\right) \vee$ acc $\left(\phi_{2}\right)$. Note that this implies that $\phi_{2}$ is false throughout $(t, u\rangle$, with the interval being right-open iff $\phi_{2}$ holds at $u$.

Let us first consider the case $b(u) \models_{\mathbb{R}} \phi_{2}$. Let $v$ be a generic instant in $(t, u)$; recall that $\phi_{2}$ is false throughout $(t, u) \supset(t, v]$. Therefore it must be $b(v) \models_{\mathbb{R}}$ $\bigcirc\left(\phi_{1}\right)$ for $b(t) \models_{\mathbb{R}} \widetilde{R}_{(0, b)}^{\downarrow}\left(\phi_{2}, \bigcirc\left(\phi_{1}\right)\right)$ to be true. So, $\phi_{1}$ holds throughout $(t, u)$ and $\phi_{2}$ holds at $u$, which means that $b(t) \models_{\mathbb{R}} \widetilde{\mathrm{U}}_{(0, b\rangle}\left(\phi_{1}, \phi_{2}\right)$.

Let us now consider the other case $b(u) \models_{\mathbb{R}} \neg \phi_{2} \wedge \widetilde{\bigcirc}\left(\phi_{2}\right)$. Let $v$ be a generic instant in $(t, u]$; recall that $\phi_{2}$ is false throughout $(t, u] \supseteq(t, v]$. From $b(t) \models_{\mathbb{R}}$ $\widetilde{\mathrm{R}}_{(0, b\rangle}^{\downarrow}\left(\phi_{2}, \bigcirc\left(\phi_{1}\right)\right)$ it must be $b(v) \models_{\mathbb{R}} \bigcirc\left(\phi_{1}\right)$. Overall, $\phi_{1}$ holds throughout $(t, u+\epsilon]$ for some $\epsilon>0$, as in particular $b(t+u) \models_{\mathbb{R}} \bigcirc\left(\phi_{1}\right)$. Clearly, this subsumes $b(t) \models_{\mathbb{R}} \widetilde{U}_{(0, b)}\left(\phi_{1}, \phi_{2}\right)$.

Finally, let $b(u) \models_{\mathbb{R}} \neg \phi_{2} \wedge \neg \widetilde{\bigcirc}\left(\phi_{2}\right) \wedge$ acc $\left(\phi_{2}\right)$. Let $v$ be a generic instant in $(t, u]$; recall that $\phi_{2}$ is false throughout $(t, u] \supseteq(t, v]$. From $b(t) \models \mathbb{R}$ $\widetilde{\mathrm{R}}_{(0, b\rangle}^{\downarrow}\left(\phi_{2}, \bigcirc\left(\phi_{1}\right)\right)$ it must be $b(v) \models_{\mathbb{R}} \bigcirc\left(\phi_{1}\right)$. Overall, $\phi_{1}$ holds throughout $(t, u+\epsilon]$ for some $\epsilon>0$, as in particular $b(t+u) \models_{\mathbb{R}} \bigcirc\left(\phi_{1}\right)$. Also, given that $\phi_{2}$ accumulates at $u$, there exists a $0<\nu<\epsilon$ such that $b(u+\nu) \models_{\mathbb{R}} \phi_{2}$. All in all, we have established that $b(t) \models_{\mathbb{R}} \widetilde{U}_{(0, b\rangle}\left(\phi_{1}, \phi_{2}\right)$.

Next, it is routine to check that the equivalences $(9-11)$ holds also when Zeno behaviors are allowed. All in all, (8-11) provide a means to replace every occurrence of strict non-matching until with a formula that contains only strict matching untils, even when Zeno behaviors are allowed. This shows that $\widetilde{\mathrm{MTL}}=$ $\widetilde{\mathrm{MTL}}^{\downarrow}$ also for Zeno behaviors.

\subsubsection{Non-Strict Variant}

Let us now prove the equivalence of the matching and non-matching variants of non-strict until. To this end, we first modify the equivalences (8-11) to deal with only non-strict operators. Correspondingly, we have the following equivalences. 


$$
\begin{aligned}
\mathrm{U}_{(0, b\rangle}\left(\phi_{1}, \phi_{2}\right) \equiv & \mathrm{U}_{(0, b\rangle}^{\downarrow}\left(\phi_{1}, \phi_{2}\right) \\
& \vee\left(\diamond_{(0, b\rangle}\left(\phi_{2}\right) \wedge \bigcirc\left(\phi_{1}\right) \wedge \mathrm{R}_{(0, b\rangle}^{\downarrow}\left(\phi_{2} \wedge \neg \phi_{1}, \bigcirc\left(\phi_{1}\right)\right)\right.
\end{aligned}
$$

Proof of Formula 24. Let us start with the $\Rightarrow$ direction, and let $t$ be the current instant. Assume that there exists a $d \in(0, b\rangle$ such that $b(t+d) \models_{\mathbb{R}} \phi_{2}$ and for all $u \in[t, t+d)$ it is $b(u) \models_{\mathbb{R}} \phi_{1}$. If also $b(t+d) \models_{\mathbb{R}} \phi_{1}$ or $b(t) \models_{\mathbb{R}} \widetilde{O}\left(\phi_{2}\right)$ then $b(t) \models_{\mathbb{R}} \mathrm{U}_{(0, b\rangle}^{\downarrow}\left(\phi_{1}, \phi_{2}\right)$, so assume that $b(t+d) \models_{\mathbb{R}} \neg \phi_{1}$ and $b(t) \models_{\mathbb{R}} \neg \widetilde{\bigcirc}\left(\phi_{2}\right)$. If also $b(t) \models_{\mathbb{R}}$ acc $\left(\phi_{2}\right)$, then there exists a $0<\nu<d$ such that $b(t+\nu) \models_{\mathbb{R}} \phi_{2}$; therefore $\phi_{2} \wedge \phi_{1}$ holds at $t+\nu \in(t, t+d) \subseteq(t, t+b\rangle$ which entails $b(t) \models_{\mathbb{R}}$ $\mathrm{U}_{(0, b\rangle}^{\downarrow}\left(\phi_{1}, \phi_{2}\right)$.

Otherwise, $\phi_{2}$ does not accumulate to the right of $t$, and thus $b(t) \models_{\mathbb{R}}$ $\widetilde{\bigcirc}\left(\neg \phi_{2}\right)$ in this case. Therefore, it is well-defined $d^{\prime}$, the smallest instant in $(t, t+b\rangle$ such that $b\left(d^{\prime}\right) \models_{\mathbb{R}} \phi_{2} \vee \widetilde{\bigcirc}\left(\phi_{2}\right) \vee \operatorname{acc}\left(\phi_{2}\right)$; note that $d^{\prime} \leq t+d$. Note that clearly $b(t) \models_{\mathbb{R}} \bigcirc\left(\phi_{1}\right)$ and $b(t) \models_{\mathbb{R}} \diamond_{(0, b\rangle}\left(\phi_{2}\right)$.

Let us first consider the case $b\left(d^{\prime}\right) \models_{\mathbb{R}} \phi_{2}$ If also $b\left(d^{\prime}\right) \models_{\mathbb{R}} \phi_{1}$ then surely $b(t) \models_{\mathbb{R}} \mathrm{U}_{(0, b\rangle}^{\downarrow}\left(\phi_{1}, \phi_{2}\right)$, so let us assume $b\left(d^{\prime}\right) \models_{\mathbb{R}} \phi_{2} \wedge \neg \phi_{1}$ without loss of generality. Let $v \in(t, t+b)$; if $v \geq t+d$, there exists a $w=t+d$ such that $w \in[t, v]$ and $b(w) \models_{\mathbb{R}} \phi_{2}$. If $v<t+d$ instead, then $v \in(t, t+d)$ so $b(v) \models_{\mathbb{R}} \bigcirc\left(\phi_{1}\right)$. All this shows that $b(t) \models_{\mathbb{R}} R_{(0, b\rangle}^{\downarrow}\left(\phi_{2} \wedge \neg \phi_{1}, \bigcirc\left(\phi_{1}\right)\right)$, which completes the proof in this case.

Let us now consider the case $b\left(d^{\prime}\right) \models_{\mathbb{R}} \neg \phi_{2} \wedge \widetilde{\bigcirc}\left(\phi_{2}\right)$; hence $d^{\prime}<t+d$. So, let us assume that $\phi_{2}$ holds over the interval $\left(d^{\prime}, d^{\prime}+\epsilon\right)$ for some $\epsilon>0$. Now, let $v \in(t, t+b\rangle$. If $v>d^{\prime}$, there exists a $w=d^{\prime}+\min \left(v-d^{\prime}, \epsilon\right) / 2$ such that $w \in[t, v]$ and $b(w) \models_{\mathbb{R}} \phi_{2}$. Again, we can assume without loss of generality that $b(w) \models_{\mathbb{R}} \neg \phi_{1}$, otherwise we would have immediately $b(t) \models_{\mathbb{R}} \bigcup_{(0, b\rangle}^{\downarrow}\left(\phi_{1}, \phi_{2}\right)$ given that $w$ is actually in $(t, v] \subset(t, t+b)$; so $b(w) \models_{\mathbb{R}} \phi_{2} \wedge \neg \phi_{1}$. If $v \leq d^{\prime}$ instead, then $v \in\left(t, d^{\prime}\right] \subset(t, t+d)$ so $b(v) \models_{\mathbb{R}} \bigcirc\left(\phi_{1}\right)$, as $\phi_{1}$ holds throughout $[t, t+d)$. All this shows that $b(t) \models_{\mathbb{R}} \mathrm{R}_{(0, b\rangle}^{\downarrow}\left(\phi_{2} \wedge \neg \phi_{1}, \bigcirc\left(\phi_{1}\right)\right)$, which completes the proof in this case as well.

Finally, assume $b\left(d^{\prime}\right) \models_{\mathbb{R}} \neg \phi_{2} \wedge \neg \widetilde{\bigcirc}\left(\phi_{2}\right) \wedge \operatorname{acc}\left(\phi_{2}\right)$; in this case $d^{\prime}<t+d$ as well. Since $\phi_{2}$ accumulates to the right of $d^{\prime}$, there exists a $0<\nu<(t+d)-d^{\prime}$ such that $b(t+d+\nu) \models_{\mathbb{R}} \phi_{2}$; therefore $\phi_{2} \wedge \phi_{1}$ holds at $t+d+\nu \in(t, t+d) \subseteq$ $(t, t+b\rangle$ which entails $b(t) \models_{\mathbb{R}} \bigcup_{(0, b\rangle}^{\downarrow}\left(\phi_{1}, \phi_{2}\right)$.

Let us now consider the $\Leftarrow$ direction, and let $t$ be the current instant. If $b(t) \models_{\mathbb{R}} \mathrm{U}_{(0, b\rangle}^{\downarrow}\left(\phi_{1}, \phi_{2}\right)$ clearly also $b(t) \models_{\mathbb{R}} \mathrm{U}_{(0, b)}\left(\phi_{1}, \phi_{2}\right)$ a fortiori. So let us assume that $\mathrm{U}_{(0, b)}^{\downarrow}\left(\phi_{1}, \phi_{2}\right)$ is false at $t$; note that this subsumes that $b(t) \models_{\mathbb{R}}$ $\neg \widetilde{\bigcirc}\left(\phi_{2} \wedge \phi_{1}\right)$ and that $b(t) \models_{\mathbb{R}} \bigcirc\left(\phi_{1}\right)$, i.e., $\phi_{1}$ holds over an interval $[t, t+\epsilon)$ for some $\epsilon>0$.

If $b(t) \models_{\mathbb{R}}$ acc $\left(\phi_{2}\right)$, then there exists a $0<\nu<\min (\epsilon, b)$ such that $b(t+\nu) \models_{\mathbb{R}}$ $\phi_{2}$; so $b(t) \models_{\mathbb{R}} U_{(0, b)}\left(\phi_{1}, \phi_{2}\right)$ a fortiori. Otherwise, if $\phi_{2}$ does not accumulate, $\widetilde{\bigcirc}\left(\neg \phi_{2}\right)$ must hold at $t$. Therefore, it is well-defined $u$, the smallest instant in $(t, t+b\rangle$ such that $b(u) \models_{\mathbb{R}} \phi_{2} \vee \widetilde{\bigcirc}\left(\phi_{2}\right) \vee$ acc $\left(\phi_{2}\right)$. Note that this implies that $\phi_{2}$ is false throughout $(t, u\rangle$, with the interval being right-open iff $\phi_{2}$ holds at $u$.

Let us first consider the case $b(u) \models_{\mathbb{R}} \phi_{2}$. Let $v$ be a generic instant in $[t, u)$; recall that $\phi_{2}$ is false throughout $(t, u) \supset(t, v]$, and that $\phi_{1}$ is true at $t$. So 
$\phi_{2} \wedge \neg \phi_{1}$ is false throughout $[t, u) \supset[t, v]$, and it must be $b(v) \models_{\mathbb{R}} \bigcirc\left(\phi_{1}\right)$ for $b(t) \models_{\mathbb{R}} \mathrm{R}_{(0, b\rangle}^{\downarrow}\left(\phi_{2} \wedge \neg \phi_{1}, \bigcirc\left(\phi_{1}\right)\right)$ to be true. So, $\phi_{1}$ holds throughout $[t, u)$ and $\phi_{2}$ holds at $u$, which means that $b(t) \models_{\mathbb{R}} \mathrm{U}_{(0, b\rangle}\left(\phi_{1}, \phi_{2}\right)$.

Let us now consider the other case $b(u) \models_{\mathbb{R}} \neg \phi_{2} \wedge \widetilde{\bigcirc}\left(\phi_{2}\right)$. Let $v$ be a generic instant in $[t, u]$; recall that $\phi_{2}$ is false throughout $(t, u] \supseteq(t, v]$, and $\phi_{1}$ is true at $t$. From $b(t) \models_{\mathbb{R}} R_{(0, b)}^{\downarrow}\left(\phi_{2} \wedge \neg \phi_{1}, \bigcirc\left(\phi_{1}\right)\right)$ it must be $b(v) \models_{\mathbb{R}} \bigcirc\left(\phi_{1}\right)$. Overall, $\phi_{1}$ holds throughout $[t, u+\epsilon]$ for some $\epsilon>0$, as in particular $b(t+u) \models_{\mathbb{R}} \bigcirc\left(\phi_{1}\right)$. Clearly, this subsumes $b(t) \models_{\mathbb{R}} \mathrm{U}_{(0, b\rangle}\left(\phi_{1}, \phi_{2}\right)$.

Finally, let $b(u) \models_{\mathbb{R}} \neg \phi_{2} \wedge \neg \widetilde{\bigcirc}\left(\phi_{2}\right) \wedge$ acc $\left(\phi_{2}\right)$. Let $v$ be a generic instant in $[t, u]$; recall that $\phi_{2}$ is false throughout $(t, u] \supseteq(t, v]$ and $\phi_{1}$ is true at $t$. From $b(t) \models_{\mathbb{R}} R_{(0, b\rangle}^{\downarrow}\left(\phi_{2} \wedge \neg \phi_{1}, \bigcirc\left(\phi_{1}\right)\right)$ it must be $b(v) \models_{\mathbb{R}} \bigcirc\left(\phi_{1}\right)$. Overall, $\phi_{1}$ holds throughout $[t, u+\epsilon]$ for some $\epsilon>0$, as in particular $b(t+u) \models_{\mathbb{R}} \bigcirc\left(\phi_{1}\right)$. Also, given that $\phi_{2}$ accumulates at $u$, there exists a $0<\nu<\epsilon$ such that $b(u+\nu) \models_{\mathbb{R}} \phi_{2}$. All in all, we have established that $b(t) \models_{\mathbb{R}} \mathrm{U}_{(0, b\rangle}\left(\phi_{1}, \phi_{2}\right)$.

$$
\begin{aligned}
& \mathrm{U}_{(a, b\rangle}\left(\phi_{1}, \phi_{2}\right) \equiv \mathrm{U}_{(a, b\rangle}^{\downarrow}\left(\phi_{1}, \phi_{2}\right) \\
& \vee\left(\square_{[0, a]}\left(\phi_{1}\right) \wedge \vartheta_{=a}\left(\mathrm{U}_{(0, b-a\rangle}\left(\phi_{1}, \phi_{2}\right)\right)\right) \\
& \mathrm{U}_{[0, b\rangle}\left(\phi_{1}, \phi_{2}\right) \equiv \phi_{2} \vee \mathrm{U}_{(0, b\rangle}\left(\phi_{1}, \phi_{2}\right) \\
& \mathrm{U}_{[a, b\rangle}\left(\phi_{1}, \phi_{2}\right) \equiv\left(\diamond_{=a}\left(\phi_{2}\right) \wedge \square_{[0, a)}\left(\phi_{1}\right)\right) \vee \mathrm{U}_{(a, b\rangle}\left(\phi_{1}, \phi_{2}\right)
\end{aligned}
$$

All in all, (24-27) provide a means to replace every occurrence of non-strict non-matching until with a formula that contains only non-strict matching untils, even when Zeno behaviors are allowed. This shows that MTL $=$ MTL ${ }^{\downarrow}$ also for Zeno behaviors.

\section{Summary and Discussion}

Let us provide a summary of the relative expressiveness results of this paper.

Figure 5 displays the relative expressiveness relations for non-Zeno behaviors. Note that the problem of the relationship between $b \widetilde{\mathrm{MTL}}^{\downarrow}$ and bMTL is still open. In particular, it may be b $\widetilde{\mathrm{MTL}}^{\downarrow} \subset b \mathrm{MTL}$, or $b \widetilde{\mathrm{MTL}}^{\downarrow} \supset \mathrm{bMTL}$, or $b \widetilde{\mathrm{MTL}}^{\downarrow}=\mathrm{bMTL}$, or even that $b \widetilde{\mathrm{MTL}}^{\downarrow}$ and $b \mathrm{MTL}$ have orthogonal expressive power.

Figure 6 displays the relative expressiveness relations if Zeno behaviors are allowed. All the separation proofs for flat formulas exploit non-Zeno behaviors; therefore they subsume the separation of the same language classes over generic (i.e., including Zeno) behaviors.

Future work. Future work will first of all try to assess the relative expressiveness relations that are still unknown. It will also consider other variations that may change the currently known relative expressiveness relations, such as the use of past operators. 


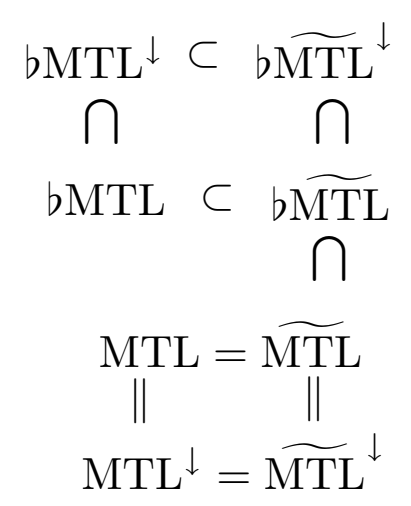

Figure 5: Expressiveness over non-Zeno behaviors.

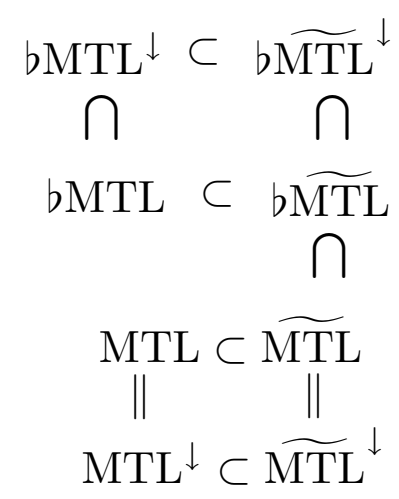

Figure 6: Expressiveness over Zeno behaviors.

Finally, it may be interesting to give a precise characterization of the expressiveness of MTL based on the nesting depth of its formulas. Previous work of this kind has been done for MITL in [AH92a], and for classical temporal logic in several works such as [EW96, TW04, KS05].

Acknowledgements. We thank the anonymous referees, especially \# 4, who reviewed the conference version of this paper [FR07b] for their scrupulous and very relevant observations that definitely contributed to improve our work.

\section{References}

[AFH96] Rajeev Alur, Tomás Feder, and Thomas A. Henzinger. The benefits of relaxing punctuality. Journal of the ACM, 43(1):116-146, 1996.

[AH92a] Rajeev Alur and Thomas A. Henzinger. Back to the future: Towards a theory of timed regular languages. In Proceedings of the 33rd Annual Symposium on Foundations of Computer Science (FOCS'92), pages 177186. IEEE Computer Society Press, 1992. 
[AH92b] Rajeev Alur and Thomas A. Henzinger. Logics and models of real time: A survey. In J. W. de Bakker, Cornelis Huizing, and Willem P. de Roever, editors, Proceedings of the Real-Time: Theory in Practice, REX Workshop, volume 600 of Lecture Notes in Computer Science, pages 74-106. Springer-Verlag, 1992.

[AH93] Rajeev Alur and Thomas A. Henzinger. Real-time logics: Complexity and expressiveness. Information and Computation, 104(1):35-77, 1993.

[AH94] Rajeev Alur and Thomas A. Henzinger. A really temporal logic. Journal of the ACM, 41(1):181-204, 1994.

[AL94] Martín Abadi and Leslie Lamport. An old-fashioned recipe for real-time. ACM Transactions on Programming Languages and Systems, 16(5):15431571, 1994.

[Asa04] Eugene Asarin. Challanges in timed languages: from applied theory to basic theory. Bulletin of the European Association for Theoretical Computer Science, 83:106-120, 2004.

[BCM05] Patricia Bouyer, Fabrice Chevalier, and Nicolas Markey. On the expressiveness of TPTL and MTL. In R. Ramanujam and Sandeep Sen, editors, Proceedings of the 25th International Conference on Foundations of Software Technology and Theoretical Computer Science (FSTTCS'05), volume 3821 of Lecture Notes in Computer Science, pages 432-443. SpringerVerlag, 2005.

[BMOW07] Patricia Bouyer, Nicolas Markey, Joël Ouaknine, and James Worrell. The cost of punctuality. In Proceedings of the 22nd IEEE Symposium on Logic in Computer Science (LICS'07). IEEE Computer Society, 2007.

[CC00] Hubert Comon and Véronique Cortier. Flatness is not a weakness. In Proceedings of the 14th Annual Conference of the EACSL on Computer Science Logic, volume 1862 of Lecture Notes in Computer Science, pages 262-276. Springer-Verlag, 2000.

[Dam99] Dennis Dams. Flat fragments of CTL and CTL*: Separating the expressive and distinguishing powers. Logic Journal of the IGPL, 7(1):55-78, 1999.

[DG99] Volker Diekert and Paul Gastin. An expressively complete temporal logic without past tense operators for Mazurkiewicz traces. In Proceedings of the 13th International Workshop on Computer Science Logic (CSL'99), volume 1683 of Lecture Notes in Computer Science, pages 188203. Springer-Verlag, 1999.

[DMP07] Deepak D'Souza, Raj Mohan M., and Pavithra Prabhakar. Flattening metric temporal logic. Manuscript, 2007.

[DP07] Deepak D'Souza and Pavithra Prabhakar. On the expressiveness of MTL in the pointwise and continuous semantics. Formal Methods Letters (Software Tools for Technology Transfer), 9(1):1-4, 2007.

[DS02] Stéphane Demri and Philippe Schnoebelen. The complexity of propositional linear temporal logics in simple cases. Information and Computation, 174(1):84-103, 2002.

[Eme90] E. Allen Emerson. Temporal and modal logic. In Jan van Leeuwen, editor, Handbook of Theoretical Computer Science, volume B, pages 996-1072. Elsevier Science, 1990.

[EW96] Kousha Etessami and Thomas Wilke. An until hierarchy for temporal logic. In Proceedings of the 11th Annual IEEE Symposium on Logic in Computer Science (LICS'96), pages 108-117. IEEE Computer Society Press, 1996. 
[FMMR07] Carlo A. Furia, Dino Mandrioli, Angelo Morzenti, and Matteo Rossi. Modeling time in computing: a taxonomy and a comparative survey. Technical Report 2007.22, Dipartimento di Elettronica e Informazione, Politecnico di Milano, January 2007.

[FR06] Carlo A. Furia and Matteo Rossi. Integrating discrete- and continuoustime metric temporal logics through sampling. In Eugene Asarin and Patricia Bouyer, editors, Proceedings of the 4 th International Conference on Formal Modelling and Analysis of Timed Systems (FORMATS'06), volume 4202 of Lecture Notes in Computer Science, pages 215-229. SpringerVerlag, September 2006.

[FR07a] Carlo A. Furia and Matteo Rossi. No need to be strict: on the expressiveness of metric temporal logics with (non-)strict operators. Bulletin of the European Association for Theoretical Computer Science, 2007. To appear.

[FR07b] Carlo A. Furia and Matteo Rossi. On the expressiveness of MTL variants over dense time. In Proceedings of the 5th International Conference on Formal Modelling and Analysis of Timed Systems (FORMATS'07), Lecture Notes in Computer Science. Springer-Verlag, 2007.

[Fur07] Carlo Alberto Furia. Scaling Up the Formal Analysis of Real-Time Systems. PhD thesis, Dipartimento di Elettonica e Informazione, Politecnico di Milano, May 2007.

[GHR94] Dov M. Gabbay, Ian Hodkinson, and Mark Reynolds. Temporal Logic (vol. 1): mathematical foundations and computational aspects, volume 28 of Oxford Logic Guides. Oxford University Press, 1994.

[GM01] Angelo Gargantini and Angelo Morzenti. Automated deductive requirement analysis of critical systems. ACM Transactions on Software Engineering and Methodology, 10(3):255-307, 2001.

[GPSS80] Dov M. Gabbay, Amir Pnueli, Saharon Shelah, and Jonathan Stavi. On the temporal basis of fairness. In Conference Record of the 7th Annual ACM Symposium on Principles of Programming Languages (POPL'80), pages 163-173, 1980.

[HR03] Yoram Hirshfeld and Alexander Moshe Rabinovich. Future temporal logic needs infinitely many modalities. Information and Computation, 187(2):196-208, 2003.

[HR04] Yoram Hirshfeld and Alexander Moshe Rabinovich. Logics for real time: Decidability and complexity. Fundamenta Informaticae, 62(1):1-28, 2004.

[HR06] Yoram Hirshfeld and Alexander Moshe Rabinovich. Expressiveness of metric modalities for continuous time. In Dima Grigoriev, John Harrison, and Edward A. Hirsch, editors, Proceedings of the 1st International Computer Science Symposium in Russia: Computer Science - Theory and Applications (CSR'06), volume 3967 of Lecture Notes in Computer Science, pages 211-220. Springer-Verlag, 2006.

[Koy90] Ron Koymans. Specifying real-time properties with metric temporal logic. Real-Time Systems, 2(4):255-299, 1990.

[KS05] Antonín Kučera and Jan Strejček. The stuttering principle revisited. Acta Informatica, 41(7/8):415-434, 2005.

[LMS02] François Laroussinie, Nicolas Markey, and Ph. Schnoebelen. Temporal logic with forgettable past. In Proceedings of the 17th IEEE Symposium on Logic in Computer Science (LICS'02), pages 383-392. IEEE Computer Society, 2002. 
[LW05] Slawomir Lasota and Igor Walukiewicz. Alternating timed automata. In Vladimiro Sassone, editor, Proceeding of the 8th International Conference on Foundations of Software Science and Computational Structures (FOSSACS'05), volume 3441 of Lecture Notes in Computer Science, pages 250-265. Springer-Verlag, 2005.

[MC85] Bhubaneswaru Mishra and Edmund M. Clarke. Hierarchical verification of asynchronous circuits using temporal logic. Theoretical Computer Science, 38:269-291, 1985.

[MNP05] Oded Maler, Dejan Nickovic, and Amir Pnueli. Real time temporal logic: Past, present, future. In Paul Petterson and Wang Yi, editors, Proceedings of the 3rd International Conference on Formal Modeling and Analysis of Timed Systems (FORMATS'05), volume 3829 of Lecture Notes in Computer Science, pages 2-16. Springer-Verlag, 2005.

[MNP06] Oded Maler, Dejan Nickovic, and Amir Pnueli. From MITL to timed automata. In Eugene Asarin and Patricia Bouyer, editors, Proceedings of the 4 th International Conference on Formal Modeling and Analysis of Timed Systems (FORMATS'06), volume 4202 of Lecture Notes in Computer Science, pages 274-289. Springer-Verlag, 2006.

[OW07] Joël Ouaknine and James Worrell. On the decidability and complexity of metric temporal logic over finite words. Logical Methods in Computer Science, 3(1), 2007.

[PD06] Pavithra Prabhakar and Deepak D'Souza. On the expressiveness of MTL with past operators. In Eugene Asarin and Patricia Bouyer, editors, Proceedings of the 4th International Conference on Formal Modeling and Analysis of Timed Systems (FORMATS'06), volume 4202 of Lecture Notes in Computer Science, pages 322-336. Springer-Verlag, 2006.

[Rab03] Alexander Moshe Rabinovich. Automata over continuous time. Theoretical Computer Science, 300(1-3):331-363, 2003.

[Rey03] Mark Reynolds. The complexity of the temporal logic with until over general linear time. Journal of Computer and System Sciences, 66(2):393426, 2003.

[TW04] Denis Thérien and Thomas Wilke. Nesting until and since in linear temporal logic. Theory of Computing Systems, 37(1):111-131, 2004. 